\title{
Distinct hippocampal network states support theta phase precession and theta sequences in CA1
}

\author{
Matteo Guardamagna ${ }^{1, \dagger}$, Federico Stella ${ }^{1, *, \dagger}$ and Francesco P. Battaglia ${ }^{1, *, \dagger}$ \\ ${ }^{1}$ Donders Institute for Brain, Cognition and Behaviour, Radboud University, Nijmegen, the Netherlands \\ * Co-senior author \\ ${ }^{\dagger}$ Correspondence matteo.guardamagna@donders.ru.nl (M.G.), federico.stella@donders.ru.nl (F.S.), \\ f.battaglia@science.ru.nl (F.P.B.)
}

\section{Summary}

The hippocampus likely uses temporal coding to represent complex memories via mechanisms such as theta phase precession and theta sequences. Theta sequences are rapid sweeps of spikes from multiple place cells, encoding past or planned trajectories or non-spatial information. Phase precession, the correlation between a place cell's theta firing phase and animal position has been suggested to facilitate sequence emergence. We find that $\mathrm{CA} 1$ phase precession varies strongly across cells and environmental contingencies. Phase precession depends on the CA1 network state, and is only present when the medium gamma oscillation $(60-90 \mathrm{~Hz}$, linked to Entorhinal inputs) dominates. Conversely, theta sequences are most evident for non-precessing cells or with leading slow gamma (20-45 Hz, linked to CA3 inputs). These results challenge the view that phase precession is the mechanism underlying the emergence of theta sequences and point at a "dual network states" model for hippocampal temporal code, potentially supporting merging of memory and exogenous information in CA1. 


\section{Introduction}

According to current theories (McNaughton et al. 2006, Buzsáki and Moser, 2013, Eichenbaum, 2017), the hippocampus combines heterogeneous information from both memory and the outside world to generate representations of episodes and complex occurrences, with space and time as the main organizing principles. These representations may then serve as index codes (Teyler and DiScenna, 1986, McClelland et al. 1995) that propagate to the entire brain, feeding detailed recall, vivid re-experiencing, mental simulation and prediction of the world's future state and planning of upcoming behavior (Squire and Knowlton, 1995, Foster and Wilson, 2006, Lisman and Redish, 2009, Carr et al., 2011, Gupta et al., 2012, Pfeiffer and Foster, 2013, Wikenheiser and Redish, 2015, Renoult et al., 2019, Wang et al., 2020). Within the hippocampus, the CA1 subfield sits at the confluence of two information streams: the first, directly arising from Layer III pyramidal cells in the Entorhinal Cortex (EC3), carries signals about the animal's position in space, and highly processed sensory information about landmarks and other relevant environmental features (Witter and Moser, 2006, Moser et al. 2008, Deshmukh and Knierim, 2011). The second, arising from the Schaffer collaterals coming from the CA3 subfield, is thought to mostly reflect retrieved memories from the auto-associative networks in that structure (Shepherd, 2004, Treves and Rolls, 1994). In CA1, these two streams are merged, and may be compared, providing a neural mechanism that allows for the updating of memories with incoming external information, and in turn, the pattern completion from memory of missing information in the external world (Vinogradova, 2001. Duncan et al., 2012).

The dynamics of the interactions between these structures is likely to shape this computational operation. Timings of the EC3 and CA3 inputs are partially segregated, as they peak at different phases of the theta rhythm, a steady $\sim 8 \mathrm{~Hz}$ oscillation ubiquitous in the hippocampal formation of active rodents (Buzsáki, 2002). Furthermore, communication from, respectively, Entorhinal Cortex and CA3 to CA1 is linked to different dynamical network states: Entorhinal inputs correlate with the emergence of medium gamma (60-90 Hz) oscillations in the stratum lacunosum moleculare (slm), whereas the CA3 contribution is associated with slow gamma oscillations $(20-45 \mathrm{~Hz})$ in the stratum radiatum (sr) (Bragin et al., 1995, Colgin et al., 2009, Lasztóczi and Klausberger 2014. Fernández-Ruiz et al., 2017). The intensity of these two oscillations changes dynamically on the scale of hundreds of milliseconds, and the coding properties of CA1 place cells, neurons activating when the animal is at precise locations in the environment, change accordingly (Cabral et al., 2014, Bieri et al., 2014). During elevated slow gamma, place representations seem to be prospective (reflecting an internally generated prediction of where the animal will be a few moments later) whereas concomitant to strong medium gamma place cell representations veer retrospective (reflecting a position in the past, and likely depending on sensory input (Cabral et al. 2014)). Together, these findings suggest that CA1 can segregate or combine information of endogenous and exogenous origin in a dynamic and flexible way, to fulfill a diversity of computational demands. To subserve such a complex function, and provide information-rich indices to the rest of the brain, the hippocampus likely makes use of temporal codes. Activity of hippocampal neural ensembles is organized in sequences, that can be replayed during short pauses in behavior or sleep (Lee and Wilson, 2002, Johnson and Redish, 2007, Davidson 
et al. 2009, Pfeiffer and Foster, 2015, Ólafsdóttir et al., 2018). Neuronal sequences also emerge at a compressed timescale in individual cycles of the ongoing theta rhythm, mirroring the order of activation at the behavioral timescale (Dragoi and Buzsáki, 2006, Foster and Wilson, 2007). Since the timescale at which theta sequences occur is compatible with spike-timing dependent synaptic plasticity rules (Markram et al., 1997), they have been deemed the main organizational mechanism for spatial and non-spatial episodic memory traces (Drieu and Zugaro, 2019). Theta phase precession, the systematic backing up in the firing phase of place cells with respect to theta, as the animal advances in the place field (O'Keefe and Recce, 1993), has been seen as a key organizing principle for hippocampal temporal codes. While the mechanisms giving rise to phase precession are still widely debated (Tsodyks et al., 1996, O’Keefe and Recce, 1993, Mehta et al., 2000, 2002, Harris et al. 2002, Yamaguchi et al., 2002, Chance, 2012), two functional roles have been generally assumed for it. First, phase precession would induce a phase coding (Huxter et al. 2003), that is, the theta firing phase of place cells would be a key conduit for information about the animal's location. Second, phase precession would be the main 'engine' behind sequence formation: under certain assumptions, the presence of phase precession implies theta sequences, that is, a millisecond-scale temporal ordering between the firing of place cells with neighboring place fields, favoring spike-timing dependent plasticity (Skaggs et al., 1996, Reifenstein et al., 2021, Chadwick et al. 2015).

Both of these functional roles, however, depend on phase precession and theta sequences taking place in a reliable, nearly noise-free fashion, and would break down under high variance conditions. Yet, while we know that phase precession patterns change on a lap-by-lap basis (Schmidt et al. 2009$)$, and that theta sequences and phase precession not always co-exist (Feng et al., 2015, Middleton and McHugh, 2016, Drieu et al., 2018), a full picture of how hippocampal temporal coding varies under the influence of the network state is not available. Here, we present a systematic analysis of the variability in phase precession and spike timing of place cells in mice. We characterize such features of neural activity change for different neurons at different spatial locations, and as a function of CA1 dynamical state, as assessed by a precise, layer-resolved determination of the instantaneous activity of the medium and slow gamma circuits. We found that, far from being a stable property of place cells, phase precession varied in degree from neuron to neuron, and for the same neuron, from one place field to another. Even for place fields with strong precession, this was only present when medium gamma dominates over slow gamma, and was virtually absent at other times. Furthermore, theta sequences only involve phase precessing cells during periods of strong slow gamma, suggesting that these two phenomena are complementary, rather than functionally linked, and depend on different network mechanisms. In fact, non-precessing place cells express theta sequences more reliably than precessing ones.

We believe that our results call for a revision of current theories on information encoding in the hippocampus. We speculate that the distinction and interplay of EC3 and CA3 inputs, the heterogeneity in the afferent connectivity to different place cells and dendritic dynamics play a bigger role than currently thought in shaping the timing of CA1 neuronal firing. While these data challenge current views on how phase precession may be the main determinant of theta sequences, the picture opening up may entail a much greater flexibility and computational power for CA1 than currently imagined. 


\section{Results}

\section{The Hybrid Drive enables large scale place cell recordings with layer-resolved os- cillations in freely moving mice}

We implanted 6 mice with the Hybrid Drive (Figure 1A; (Guardamagna et al., 2021)), a novel recording device that combines linear silicon probes with high density tetrode arrays. Tetrodes were individually lowered to target pyramidal cell bodies in the stratum pyramidale of the CA1 region (Figure 1 B,C) and enabled us to collect large ensemble activity of pyramidal neurons (up to $\approx 100)$ for up to 10 days. The vertical span $(960$ $\mu \mathrm{m}$ ) of the silicon probe yielded LFPs from all CA1 layers (Figure $1 \mathrm{C}, \mathrm{G}$ ).

This combination of within layer and across-layer recording techniques enabled us to obtain a layer-resolved picture of hippocampal local field potentials (LFPs) (Figure 1G; (Lasztóczi and Klausberger, 2014)), while simultaneously monitoring the activity of large populations of place cells (Figure $1 \mathrm{H}$; (Lasztóczi and Klausberger. 2016)) as animals alternated runs between the two ends of a linear track (Figure 1D).

Over the course of 10 consecutive days, mice learned to collect rewards at both ends of a 1 meter-long linear track. While mice were never food or water deprived, we nevertheless observed that performance quickly increased in the first few days and then improved steadily, as measured by the number of laps completed on each day (Figure 1F). For analysis, we considered data from the two running directions separately, by dividing left-to-right from right-to-left runs (Figure $1 \mathrm{E}$, violet and light blue, respectively). Among pyramidal neurons we selected those with significant spatial modulation (see Place Cell Identification). These place cells exhibited place fields that tiled the whole linear track in both running directions (Figure $1 \mathrm{H}$ ). To investigate properties of stable, well-learned hippocampal spatial representations, we focused on the data obtained from the later recording sessions (between day 7 and 10 of training), which also were the most consistent in terms of number of laps and velocity profiles (Figure $1 \mathrm{~F}$ ). 
bioRxiv preprint doi: https://doi.org/10.1101/2021.12.22.473863; this version posted December 23, 2021. The copyright holder for this
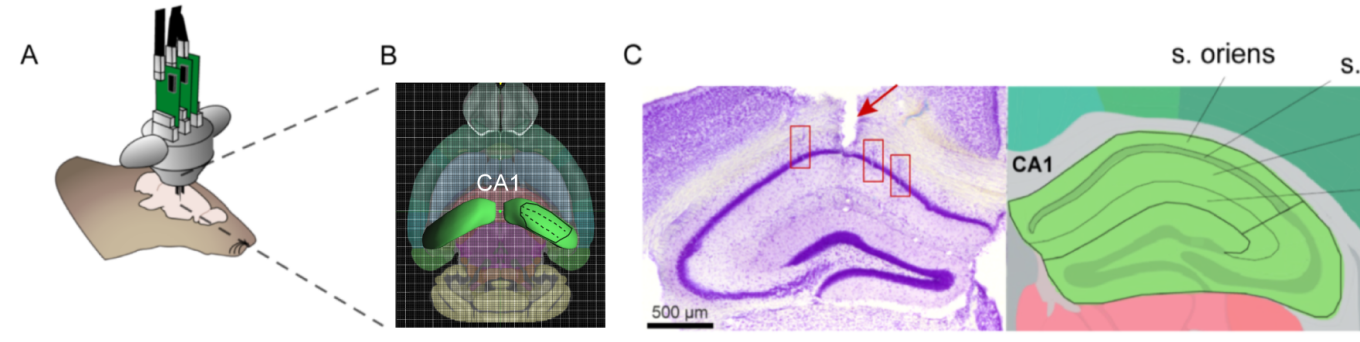

s. pyramidale

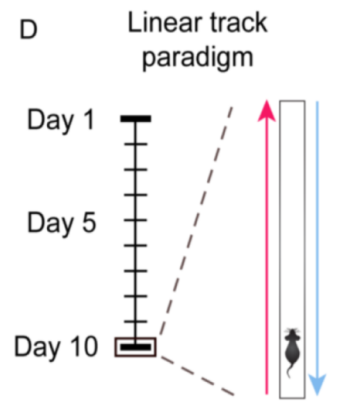

E

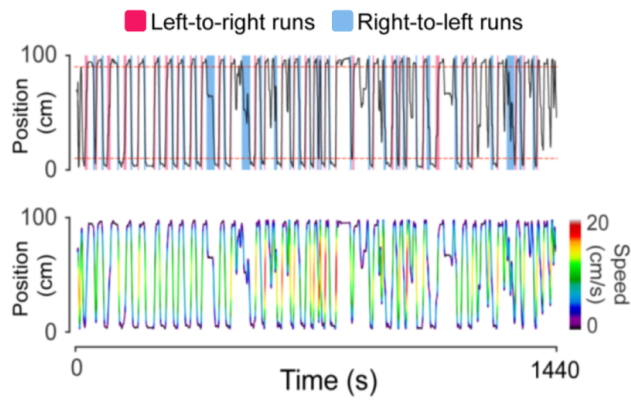

F

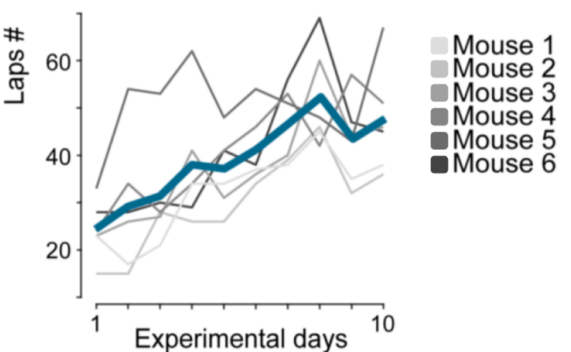

G

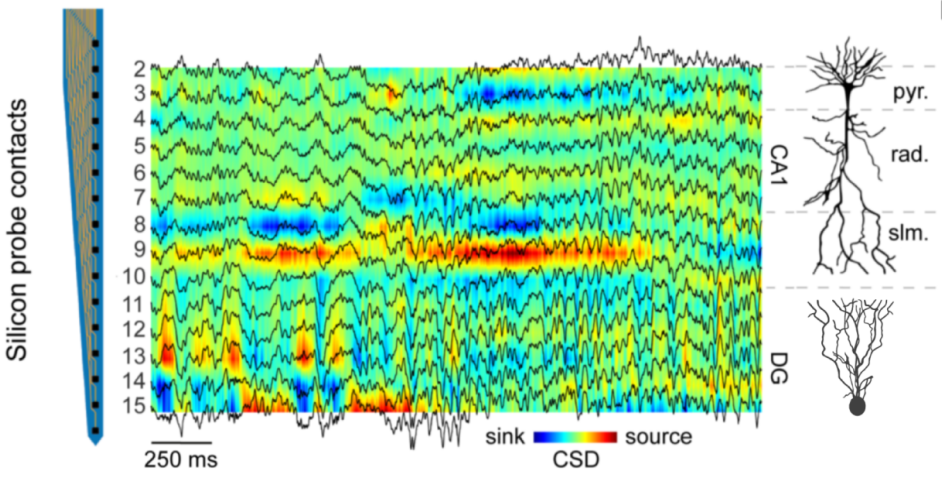

$\mathrm{H}$

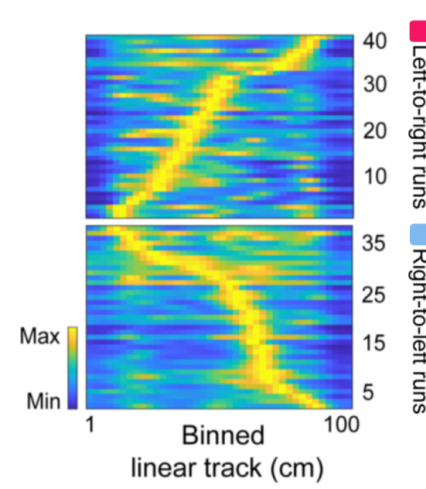

$\frac{0}{00}$
0
0
$\frac{8}{0}$
$\frac{D}{\omega}$
$\#$

Figure 1: Simultaneous recordings of CA1 place cells and layer resolved oscillations during goal oriented behavior in freely-moving mice. (A) Illustration of the Hybrid Drive implanted on a mouse. (B) Image from the Allen Brain Explorer (beta), a 3D volumetric reference atlas of the mouse brain. Image credit: Allen Institute. The dorsal CA1 region of the hippocampus is highlighted in green. The bold black lines estimate the coverage of the implant array across the distal, intermediate and proximal sub-regions of CA1 . (C) Left: representative histology image from an example animal (Mouse 6). Red rectangles highlighted the tetrode tracks, reaching the pyramidal layer. The red arrow points to the silicon probe entry point. Right: Adapted image from the 2D coronal reference atlas, all CA1 layers. Image credit: Allen Institute. (D) Schematics of the linear track paradigm. 10 consecutive days of training without food or water deprivation. (E) Example of a linear track session from late training days (Day 10). Top: directions of motion are highlighted in two different colors (light pink: up; light blue: down). Bottom: velocity profiles are superimposed on the each lap of the session. (F) Behavioral performance of all animals across the 10 days of linear track training. Average performance is highlighted in dark blue. (G) Color coded depth profile of current sink and sources. LFP traces are superimposed in black. Silicon probe contacts (indicated on the left) span all CA1 layers and partially reach the Molecular Layer (ML) and the Granular Cell Layer (GCL) of the Dentate Gyrus (DG). (H) Example place field maps (same session as in Figure 1G). Each row represents the spatial firing of one cell along the length of the track. Firing rate in color coded and scaled to the peak firing rate for each cell. Cells are sorted according to the position of peak spatial firing on the linear track. Place field maps for both running directions are showed. 


\section{Fine-scale temporal organization of place cell activity within theta rhythm}

Theta oscillations shape the temporal organization of the firing of hippocampal neurons in many ways. There are two simplest models that may account for variability in the data: first, each neuron will fire preferentially around a certain theta phase, i.e. it is phase locked. Second, place cells will display a phase precession pattern as discussed above (Figure 2A). We found high degrees of variability for these two properties across cells and even, for the same cell, depending on the animal location on the track. Thus, cells showed varying degrees of phase locking and phase precession, and these properties are place dependent.

To fully characterize this variability, for each cell we computed a Theta Score, defined as the difference between circular-linear correlation between theta phase of firing and animal location (a common measure for phase precession (Kempter et al. 2012) $)$ and Rayleigh Vector length (estimate for phase locking). This Theta Score places each place cell on a spectrum and enables us to study how phase locking and precession properties are distributed in populations of place cells. We consistently observed Theta Scores to have an unimodal, Gaussian-like distribution across place cell populations (Figure 2B). Cells at the two ends of the interval presented good approximation of either perfect phase locking or phase precession, but most of the population could be described as a mixture of varying degree of the two modalities (Figure 2 $\mathrm{B}$ ). Theta-score appeared to be largely independent of other properties of place cells: only a very weak correlation was found with the cell Skaggs information (Figure 2D; Spearman's rho $=0.096 ; \mathrm{p}<0.01$ ), while no correlation was found with maximum firing rate (Figure 2 $\mathrm{E}$; Spearman's rho $=-0.001 ; \mathrm{p}>0.05$ ), place field size (Figure 2F; Spearman's rho $=-0.017 ; \mathrm{p}>0.05$ ) and place field activity baricenter (Figure $2 \mathrm{G}$; Spearman's rho $=0.027 ; \mathrm{p}>0.05$ ) Next, we investigated how the locking/precessing properties of a cell vary on the track and how they interact with the mean firing rate at a given location, to provide a picture of the interplay between place cells' phase and rate coding (( Huxter et al. 2003) $)$. As a location dependent measure of phase precession and locking, we took the spatial derivative of the cell's preferred firing theta phase along the track (DeltaPhi). As many cells displayed multiple place fields along the track (here, we consider runs in the two running directions separately), by comparing Delta Phi and the location-based average firing rate (FR) we can evaluate the degree of phase precession on a field-by-field basis. In this framework, "phase precessing" fields are described by consistently negative values of DeltaPhi (Figure $2 \mathrm{C}$, top row), whereas "phase locking" place fields display near zero (or positive) DeltaPhi (bottom row examples in Figure 2C). Similarly to the Theta Score, each cell activity relationship with the phase of theta can be summarized by computing the correlation along the track between the cell firing rate and the associated DeltaPhi (phase precessing cells being expected to have an inverse relationship between firing rate increases and negative deflection of the preferred phase of spikes). This measure and the Theta Score are highly correlated (Figure S3, Spearman's rho $=-0.175 ; \mathrm{p}<0.05$ ). 
bioRxiv preprint doi: https://doi.org/10.1101/2021.12.22.473863; this version posted December 23, 2021. The copyright holder for this preprint (which was not certified by peer review) is the author/funder, who has granted bioRxiv a license to display the preprint in perpetuity. It is made available under aCC-BY-NC-ND 4.0 International license.

\section{A}

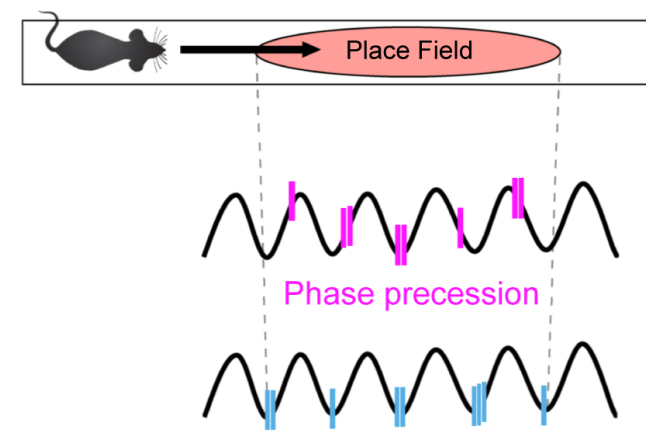

Phase locking

C
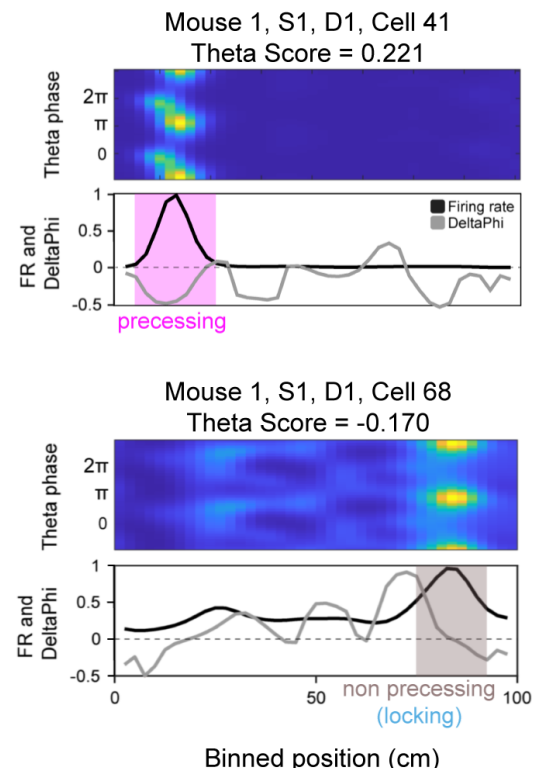
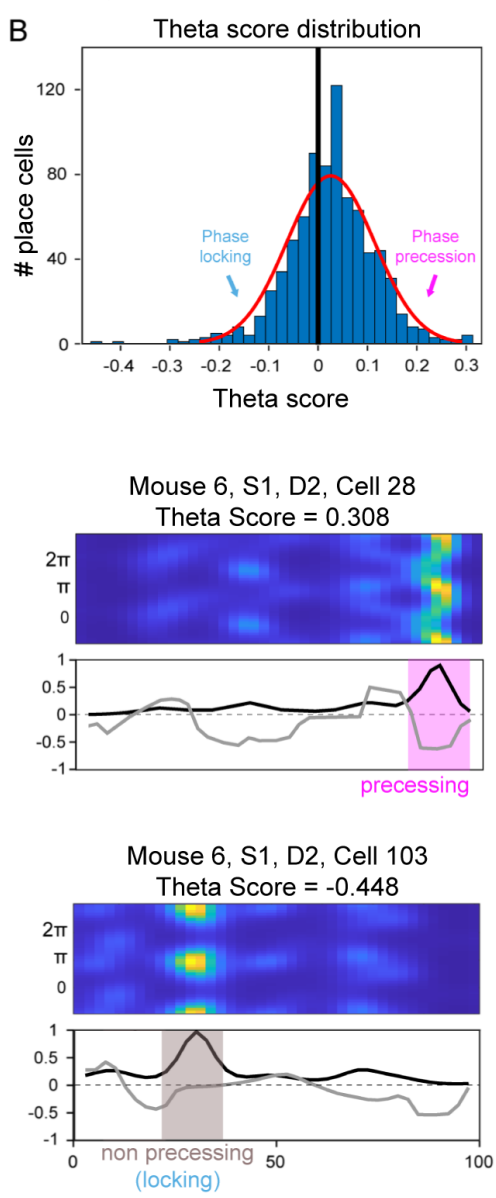

Binned position $(\mathrm{cm})$
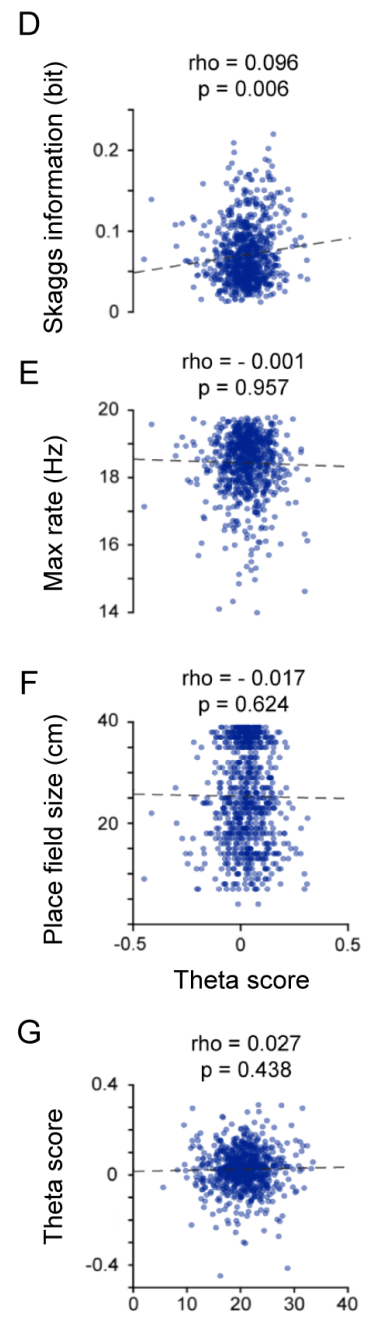

Activity baricenter $(\mathrm{cm})$

Figure 2: CA1 place cells relationship to the theta rhythm is characterised by a multi-phase nature (A) Visualization of the two modes of place cells' temporal organization to theta. Phase precession is described as the systematic advancement of spiking phases as the animal traverses the place field. Phase locking describes the tendency of firing at the same phase of the underlying oscillation. (B) Theta Score distribution of all recorded place cells across all sessions (11) and all animals (6). (C) Examples from individual place cells, from different portions of the Theta Score distribution. Top to bottom: Color-coded spatiotemporal maps showing the mean firing rate (colour) as a function of position $(\mathrm{x})$ and phase $(\mathrm{y})$; linearized firing rate $(\mathrm{FR})$ maps in solid black lines and phase-position maps (DeltaPhi) in light-gray lines. (D) Scatterplot of Theta Score values versus Skaggs information measure (bits), for field one of all recorded place cells (Spearman's rho $=0.096 ; \mathrm{p}<$ 0.01) (E) Scatterplot of Theta Score values versus max firing rate (Hz), for field one of all recorded place cells (Spearman's rho $=-0.001 ; \mathrm{p}>0.05)$. (F) Scatterplot of Theta Score values versus place field size $(\mathrm{cm})$, for field one of all recorded place cells (Spearman's rho $=-0.017 ; \mathrm{p}>0.05$ ). (G) Scatterplot of activity baricenter $(\mathrm{cm})$ versus Theta Score, for field one of all recorded place cells (Spearman's rho $=0.027 ; \mathrm{p}>0.05$ ).

\section{Place cells phase coding properties vary across fields}

Phase coding modalities are not an intrinsic property of place cells, but rather have a high spatial dependence. In fact, when comparing multiple place fields from the same cell, we found that their phase coding profiles were largely independent from one another (Figure $3 \mathrm{~A}$ ). The probability to precess of field one (described as the field with the highest peak firing rate; see Place Cell Identification) was only weakly (albeit significantly) correlated (Spearman's rho $=0.154 ; \mathrm{p}<0.05)$ to the probability to precess of the secondary field of the same cell (Figure 3B; see Figure $\mathrm{S} 4$ for spike sorting information for the example neurons). Examples from single-cells reveal how 
bioRxiv preprint doi: https://doi.org/10.1101/2021.12.22.473863; this version posted December 23, 2021. The copyright holder for this preprint (which was not certified by peer review) is the author/funder, who has granted bioRxiv a license to display the preprint in perpetuity. It is made available under aCC-BY-NC-ND 4.0 International license.

the DeltaPhi measure of field one reflects a phase precessing pattern, while the spikes of the secondary field exhibit a non-precessing relationship, and vice-versa (Figure $3 \mathrm{C}$ ). Similarly, such phase coding granularity was even more strongly expressed when we considered place cells having place fields in both running directions of the track (Figure 3D). The tendency to precess of a field in one running direction was found to be statistically independent from the phase relationship of the field in the other direction (Figure $3 \mathrm{E}$; Spearman's rho $=0.092$; $\mathrm{p}>0.05)$. The propensity of single fields to precess can be thus conceived as a map-specific property, not unlike the peak firing rate and place field size (Eliav et al., 2021).

A

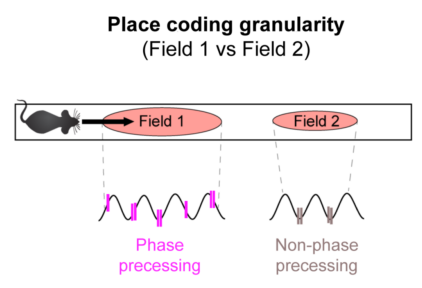

C

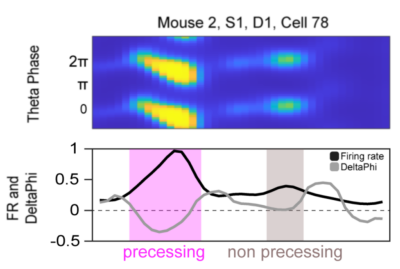

Mouse 5, S2, D1, Cell 61

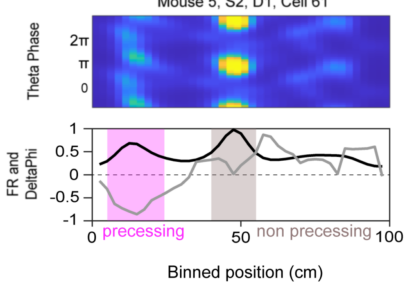

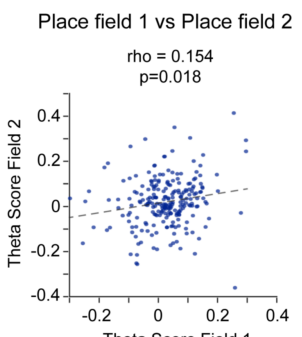

Mouse 1, S1, D2, Cell 9

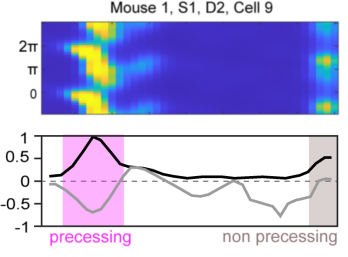

Mouse 3, S2, D1, Cell 13
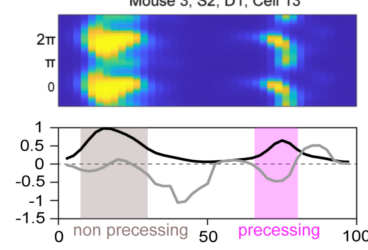

Binned position $(\mathrm{cm})$

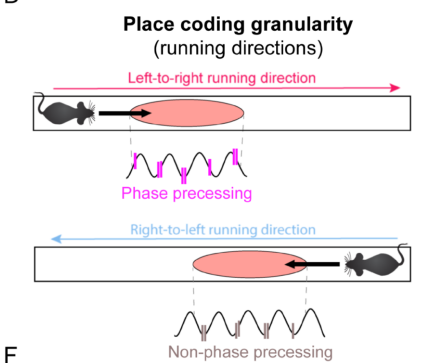

Left-to-right Mouse 1, S2, Cell 20
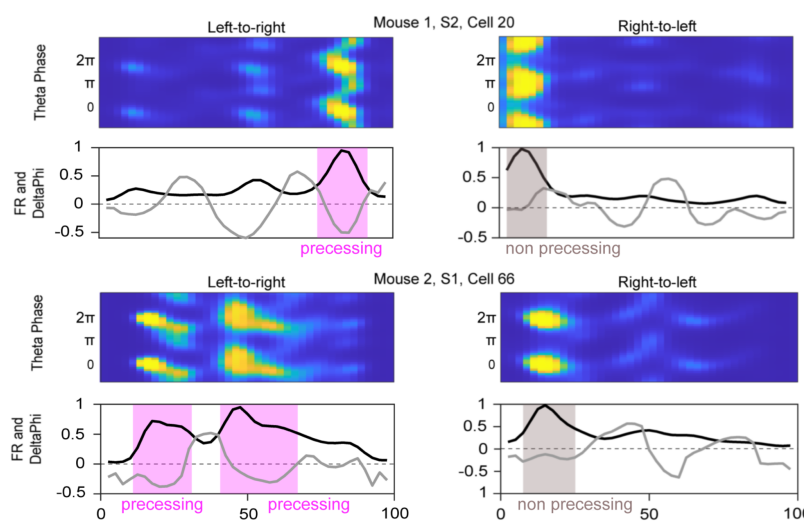

Binned position (cm)
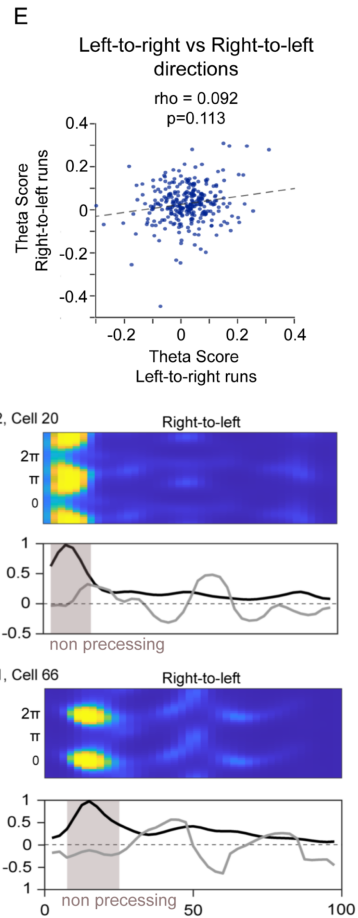

Binned position (cm)

Figure 3: Phase coding granularity between fields and running directions. (A) Schematic representation of independent phase codes (phase coding granularity) in field one and field two. (B) Scatterplot of Theta Score values of field one versus field two, for all recorded place cells with at least two fields in the same running direction (Spearman's rho $=0.154 ; \mathrm{p}>0.01$ ). (C) Examples from individual place cells. Field one expresses a phase precession pattern while the spikes of field two have a non-phase precessing relationship (top rows) and vice-versa (bottom rows). (D) Schematic representation of independent phase codes (phase coding granularity) in the place fields in the two running directions. (E) Scatterplot of Theta Score values of the field in one running direction (left-to-right) versus the field in the other running direction (right-to-left), for all recorded place cells with two place fields in the both running direction (Spearman's rho $=0.092 ; \mathrm{p}>0.05$ ). (F) Examples from individual place cells. The same place cell is expressing a phase precession pattern in one running direction, while in-field phase precession is absent in the other running direction (same session). 


\section{Current source density analysis reveal layer specific theta-gamma interaction in dorsal CA1}

In order to unfold the temporal organization of different input streams in the CA1 circuit, and their local integration, we first sought to obtain a clear picture of the interactions of theta and gamma oscillations in the network.

Current Source Density analysis (CSD; (Nicholson and Freeman, 1975, Pettersen et al., 2006, Buzsáki et al. 2012, Quiroga and Panzeri, 2013, Schomburg et al. 2014) is a standard tool to estimate the contribution of local circuits to the LFP signal and enables precise layer-level resolution, which, in the hippocampal CA1 maps neatly to different afferent pathways and groups of cells. For each CSD-time series we then computed the phase-amplitude relationship between the theta oscillation $(6-10 \mathrm{~Hz})$ and the power of oscillations at higher frequencies (15-250 Hz). Since theta power is maximal in the deeper portions of CA1 (Buzsáki, 2002, Buzsáki et al. 2003) and theta oscillation presents a high degree of coherence across layers, we decided to use the signal from the slm probe contact as the reference theta oscillation signal. This signal was then used to produce a depth-specific profile of frequency-resolved gamma-theta couplings. Note that slm theta is 180 degrees shifted from pyramidal layer theta, used as a reference in some studies.

Following Lasztóczi and Klausberger (2014) we characterized gamma oscillations in a tridimensional 'gamma space' with anatomical depth, frequency, and preferred theta phase as dimensions. Significant components in this space were then defined as contiguous regions of above-mean correlation strength. Once points in gamma space with significant coupling values found at different depths were concatenated, we obtained a 3-dimensional structure that represents theta-gamma interactions (Figure 44). We then isolated individual volumes of significant coupling with consistent spatial extension and termed them 'Basins'. These Basins provide a data-driven definition of independent gamma oscillators in the CA1 circuit. We found 3 of these Basins, coinciding with 3 separate ranges of gamma: $20-45 \mathrm{~Hz}, 60-90 \mathrm{~Hz}$ and $100-150 \mathrm{~Hz}$. In the following they are identified as low (Figure 4B), medium (Figure $4 \mathrm{C}$ ) and fast gamma (Figure $4 \mathrm{D})$ ), respectively. Each component can be characterized by its distinctive depth profile, most evident in the location of maximal coupling with theta phase (Figure $4 \mathrm{~F}$ ). While slow gamma (Figure $4 \mathrm{~B}$ ) showed minimal variability along the CA1 depth profile, with a mostly constant theta coupling strength across CA1 layers (Figure $4 \mathrm{~F}$ ), we could observe a gradual shift in the preferred coupling theta phase from more superficial layers (pyramidal) to deeper ones (slm) (Figure $4 \mathrm{E}$ ). On the contrary, the medium gamma component (Figure 4C) displayed a spatially localized pattern, with coupling strength peaking in the slm (Figure $4 \mathrm{~F}$ ). Different from slow gamma, medium gamma theta phase preference shifts abruptly between the pyramidal layer and the stratum radiatum, and stays constant in the deeper layers (Figure $4 \mathrm{E}$ ). Finally, the fast gamma component (Figure 4D) emerges only in proximity of the pyramidal layer, maintaining a constant phase preference value (Figure $4 \mathrm{E}$ ).

Based on this detailed description and visualization of gamma generators provided by the Basins, we then set out to disentangle the contributions of these circuit elements to the spike timing of place cell ensembles, and the related computations. 
A

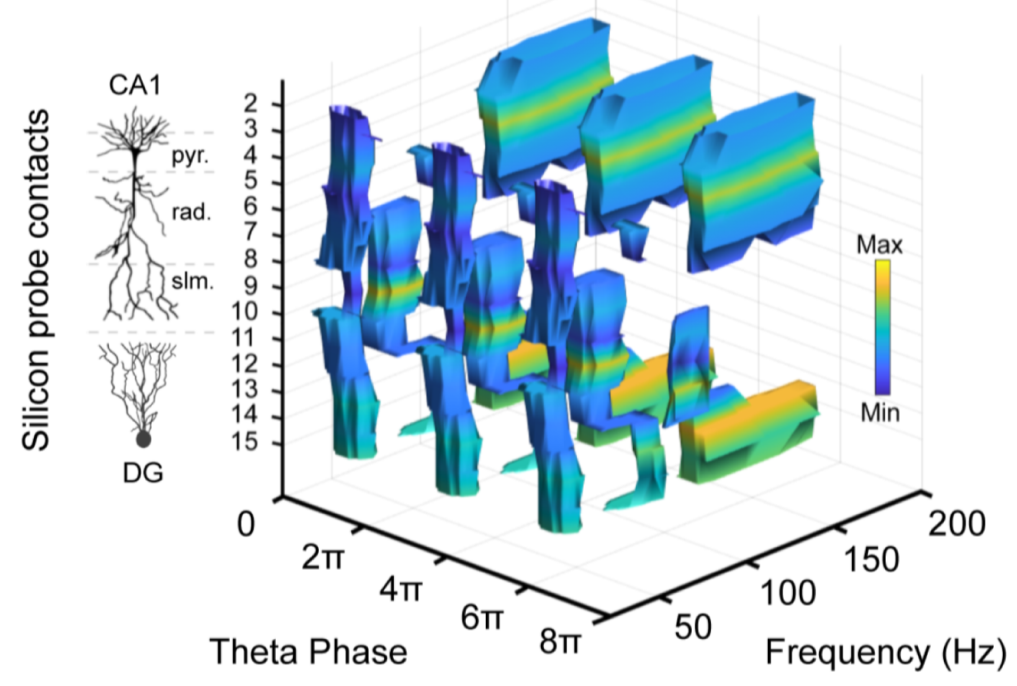

B

Slow gamma $(20-45 \mathrm{~Hz})$ component

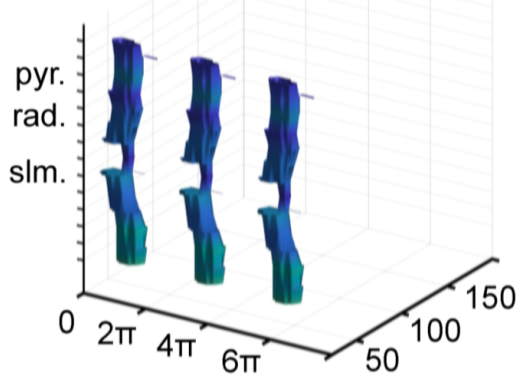

$\mathrm{E}$

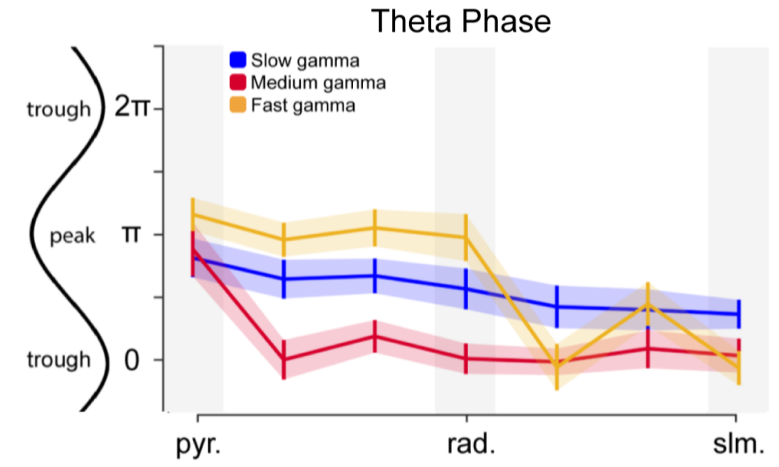

C

Medium gamma $(60-90 \mathrm{~Hz})$ component

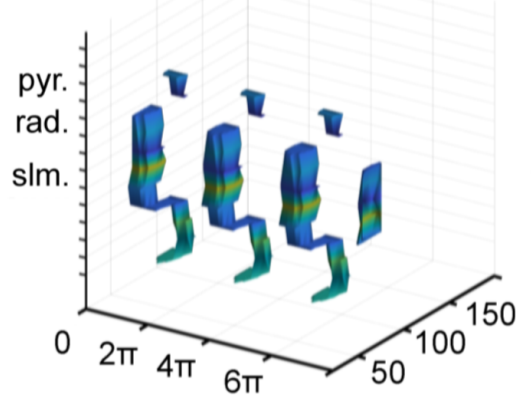

$\mathrm{F}$
$\mathrm{D}$

Fast gamma $(100-180 \mathrm{~Hz})$ component

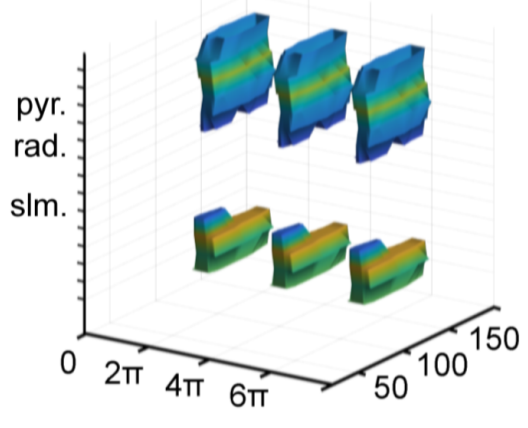

Silicon probe contacts

Figure 4: Theta-gamma interactions in a 3D phase-amplitude-depth structure. (A) CSD-derived Basins profiles, along one shank (16 recording sites; spaced at $60 \mu \mathrm{m})$ spanning the entire CA1 region (2-10) and part of the Dentate Gyrus (12-15). The Basin structure is repeated across 3 theta cycles. (B) Isolated slow gamma (20-45 Hz) Basins component from panel (A). (C) Isolated medium gamma (60-90 Hz) Basins component from panel (A). (D) Isolated fast gamma (100-180 Hz) Basins component from panel (A). (E) Theta phase preference profiles of the three Basins components across all CA1 layers. (F) Coupling strength profiles of the three Basins components across all CA1 layers. 


\section{Phase precession arises in a subset of place cells when medium gamma dominates the CA1 network state}

Because place cells do not appear to have consistent phase coding properties, we speculated that their temporal coding modalities may be affected by variability in the inputs to CA1, reflecting changing environmental conditions. We therefore investigated how different gamma regimes, considered proxies for the influence of those different input sources (Colgin et al., 2009, Lasztóczi and Klausberger, 2016), control the organization of the phase precession pattern within individual place fields. We used a Generalized Linear Model (GLM) to describe the spike probability of each place cell given i) the position within the place field; ii) the instantaneous theta phase and iii) the instantaneous power in a specific gamma range and layer (as they were obtained from the previous Basins analysis). Depending on the Theta Score values of field one (described as the field with the highest peak firing rate; see Place Cell Identification, we divided the place cell population in three equally distributed groups. Intermediate cells occupy the center of the Theta Score distribution, while phase precessing and phase locking the extremes. Crucially, we found that cells within the phase precessing population, were not performing phase precession in every theta cycle, but rather that phase precession could be seen to gradually appear in association with the increasing presence of medium gamma (relatively to slow gamma power) in the theta cycle. The spike density plot in Figure 5A reveals how the phase precession pattern gradually emerges as medium gamma becomes more dominant in the CA1 network state. The contour plots of the spike density distributions show how the appearance of spikes in the early portion of the theta cycle is conditioned to the increase of medium gamma power (Figure 5B). Conversely, changes in gamma balance do not affect the spike distributions of the phase locking and intermediate population. This is replicated for each animal separately (Figure S6. Importantly a further analysis using velocity as an additional factor in the GLM, shows that the observed gamma modulation of the phenomenon of phase precession is independent of changes in running speed of the animal (Figure S88). Spike density plots represent an average over the three sub-populations. When looking at single cell cases, the effect is still present. With increasing dominance of medium gamma, cells with a higher Theta Score tend to acquire a steeper slope (Figure 5C). This medium gamma related effect is only visible for cells with higher Theta Score, while slow gamma does not modulate the phase-position slope and the Theta Score. Once again this is replicated for each animal separately (Figure S7). Together, these observations suggest that phase precession selectively arises in a subset of place cells, as a function of the oscillatory state of the CA1 network. 
bioRxiv preprint doi: https://doi.org/10.1101/2021.12.22.473863; this version posted December 23, 2021. The copyright holder for this preprint (which was not certified by peer review) is the author/funder, who has granted bioRxiv a license to display the preprint in perpetuity. It is made available under aCC-BY-NC-ND 4.0 International license.

A

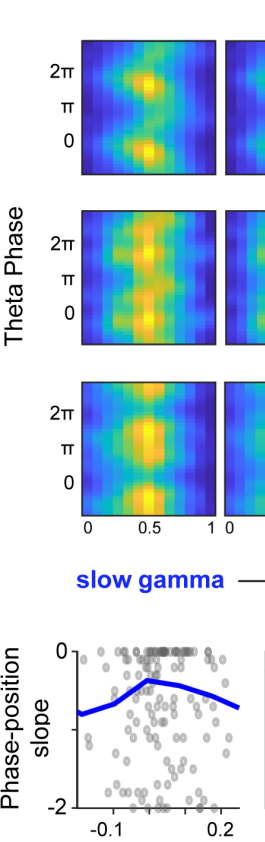

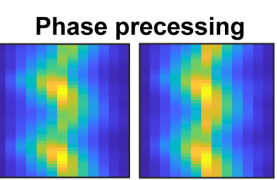

Intermediate

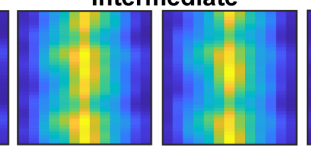

Phase Locking

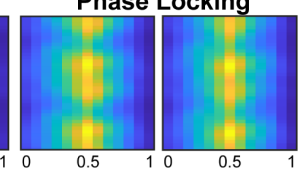

In-field position

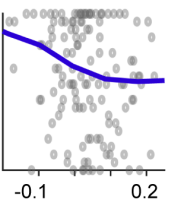

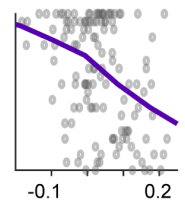

Theta Score
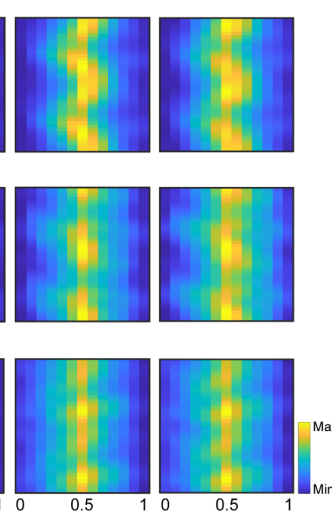

medium gamma
B
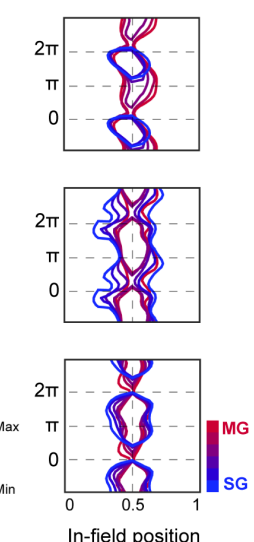

D

Phase locking Intermediate Phase precessing

$\mathrm{E}$
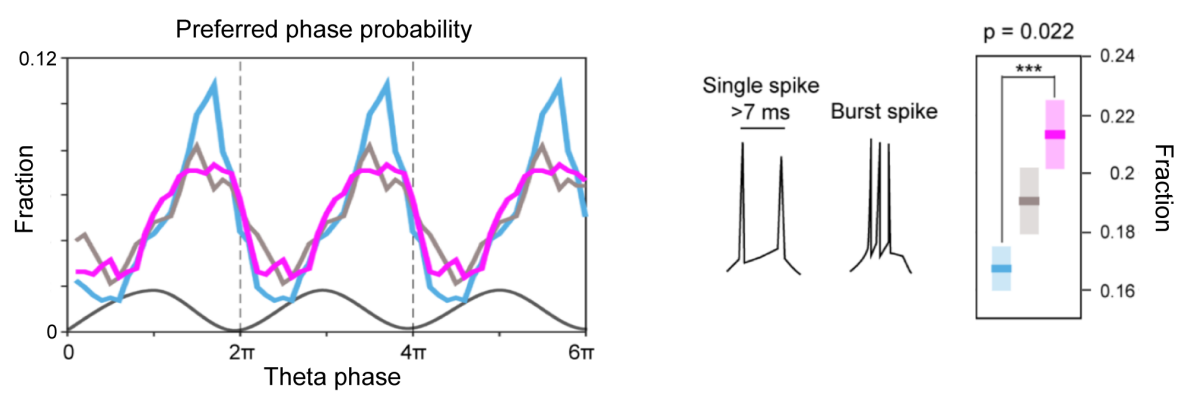

$\mathrm{F}$
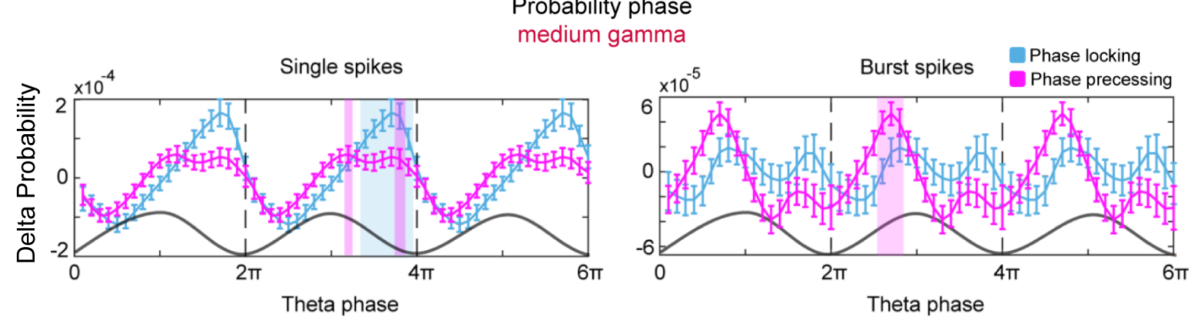

Figure 5: Phase precession arises in a sub-population of place cells during periods of medium gamma dominance (A) Spike density plot of all cells from one example animal (Mouse 1). Global average of the in-field spike probability, plotted as a function of the instantaneous gamma coefficient, for each subset of place cells (precessing, intermediate, and locking). The phase precession pattern gradually emerges only in the phase precessing subgroup, with increasing medium gamma power. (B) Contour plot of the same Spike density plot in (A), highlighting the progressive, selective emergence of a phase-location dependence for precessing cells (in association with medium gamma dominance). (C) Phase-position slope values plotted against the Theta Score values for each individual cell, as a function of slow-medium gamma balance. With increasing dominance of medium gamma, only cells with a higher Theta Score tend to acquire a steeper slope. Slow gamma has no effect on measured slope and Theta Score. (D) Preferred firing phase preferences for phase precessing, intermediate and phase locking cells. (E) Left panel, illustration of the difference between a single spike and a burst spike. Right panel, burst fraction in the phase precessing, intermediate and phase locking populations $(\mathrm{p}=0.022$, two-sample t-test). (F) Phase distribution of single spikes and burst spikes during medium gamma dominated periods, for phase locking (light blue) and phase precessing (violet) cells. Shaded areas highlight statistically significant points for positive probabilities of phase locking (light blue) and phase precessing cells (violet) $(\mathrm{p}<0.05$; Two-sample t-test). 
Dendritic dynamics might play a key role here. Long dendritic depolarizations (Gasparini et al. 2004 Mizuseki et al., 2009, Bittner et al., 2015) may induce bursts of spikes in CA1 pyramidal cells, and it is therefore interesting to explore whether bursting dynamics differs between precessing and locked cells. To clarify this point, we first assessed the preferred firing phase preferences of the phase precessing and phase locking populations. While all cells from every group similarly clustered around the descending phase of the theta phase, the only difference between groups could be found in a slightly broader preferred phase distribution for cells in the phase precessing group (Figure 5D). We then considered separately spikes that where either emitted in bursts or in isolation (ISI $>7 \mathrm{~ms}$ )(Figure $5 \mathrm{E}$, left panel). Here we found a significant modulation of burst propensity by Theta Score, with phase precessing cells having on average an higher burst fraction (Figure $5 \mathrm{E}$, right panel; $\mathrm{p}<0.05$, two-sample t-test). The higher amount of burst events, moreover, was not evenly distributed over the theta cycles, but appeared to concentrate at the earliest phases of the cycle, ahead of the phase interval containing most single spikes (Figure 5F) ( $\mathrm{p}<0.01$; Two-sample t-test).

\section{Theta sequences are increasingly more present as slow gamma dominates the CA1}

\section{network state}

Until now, we have been looking at phase coding, a potential way for single neurons to convey spatial information in addition to what is already contained in the firing rates ("Huxter et al. 2008$)$. Yet, a key role that theoretical models ascribed to phase precession is the shaping of the activity of place cells with nearby fields into temporal sequences spanning one theta cycle (Skaggs et al., 1996). Because we observed an effect of gamma oscillations on phase precession, we therefore asked whether such theta sequences were likewise affected by changes in medium and slow gamma power. We first addressed pairwise co-activation patterns, a basic, but sensitive index of sequential activation (Skaggs and McNaughton, 1996, Dragoi and Buzsáki, 2006, Middleton and McHugh, 2016). Similar to (Dragoi and Buzsáki, 2006, Middleton and McHugh, 2016), we measured the correlation between the distance between the place fields of a place cell pair and their average theta phase difference (Figure 6A). This correlation measure reflects how much a spatial distance could be reliably inferred from a temporal one. We repeated this procedure by further separating spikes according to the gamma dominating the theta cycle in which they were emitted. Measuring the gamma-dependent spatio-temporal correlation between spike pairs lead to two results. First, cells characterized by a strong phase locking showed a significant degree of spike ordering, independent of the gamma configuration at the time of the spike emission. Second, phase precessing cells, on the contrary, presented a variable amount of temporal ordering, depending on the slow-medium gamma balance. Crucially, spatio-temporal correlation of spike pairs was only present in coincidence with strong slow gamma periods, that is, only at times when phase precession was at its weakest (Figure 6B-C). Therefore place cells ability to carry information about macro-scale ordering of place fields within the micro-scale arrangement of spikes at the tens of milliseconds scale differ across cells, and depends on the current state of the networks generating gamma in CA1. Importantly, the states of these gamma networks conducive to phase precession and to pairwise ordering of spikes appeared to be complementary to one another. Pairwise interactions between cells 
provided a first approximation to evaluate the degree of which neural activity in CA1 place cells populations is sequentially organized. We took this analysis a step further by implementing a decoding procedure, aimed at estimating the level of sequential information contained in the entire population of recorded place cells. By dividing each theta cycle into a set of small sub-windows, we computed the likelihood of the activity contained in each of them to encode one of the positions of the track (6D). Using these probability density matrices, we evaluated the degree of non-locality of the encoded positions, that is to what degree the encoded positions within a theta cycle a) reflected the current position of the mouse on the track (Figure 6 $\mathrm{D}$ violet lines) or b) formed a "theta sequence", that is, they represented a set of positions sequentially distributed in time around the position of the animal (Figure 6P green lines). Again, such computation was repeated separately using spikes from either the entire population, or from specific groups of cells and also by subdividing theta cycles according to the corresponding slow-medium gamma power balance. Results obtained with Bayesian decoding confirmed and expanded those based on pairwise measures. Sequential, non-local spatial information was found to be more likely expressed during periods of slow gamma prevalence and to be more consistently present in the activity of phase locking cells (Figure 6 $6 \mathrm{E}-\mathrm{I}$ ). In fact, similarly to pairwise ordering, the information content of phase precessing cell activity was found to be modulated by gamma. While with medium gamma they produced a higher degree of coding of the current animal position, this progressively shifted towards fast advancing theta sequences with increasing slow gamma (Figure 6G). No modulation was observed for phase locking cells (Figure 6F). Finally, the coding properties of spikes emitted at different portions of the theta cycles resulted to be non-uniform in medium gamma dominated periods. Indeed, by applying our analysis to the first and the second half of the theta cycle separately, we found that sequential encoding by phase locking cells was mostly found in the later stages of the theta cycles, while in the first part phase precessing and phase locking cells behaved in a very similar way and both encoded non-local information (Figure 6[). Consistently with our previous analysis, thus, the population approach confirmed that phase precession and sequential coding emerged in CA1 under different network conditions. 
bioRxiv preprint doi: https://doi.org/10.1101/2021.12.22.473863; this version posted December 23, 2021. The copyright holder for this

A

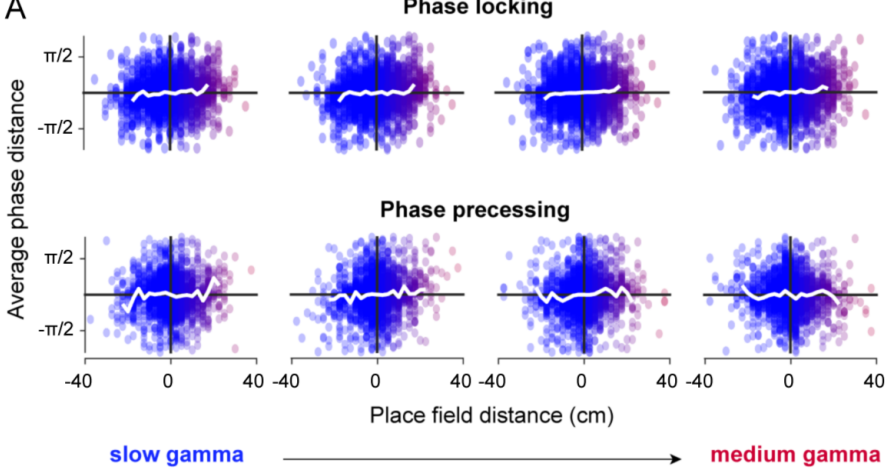

B

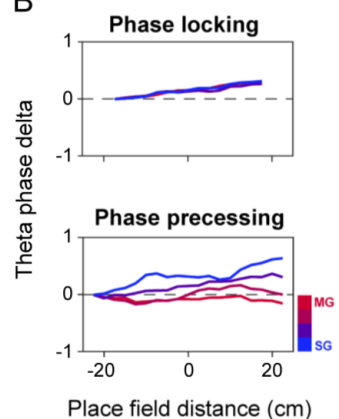

C

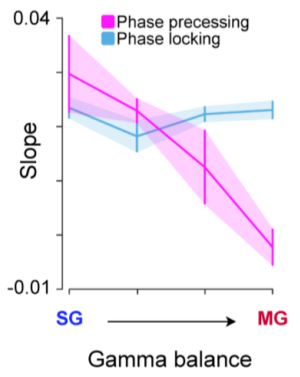

D
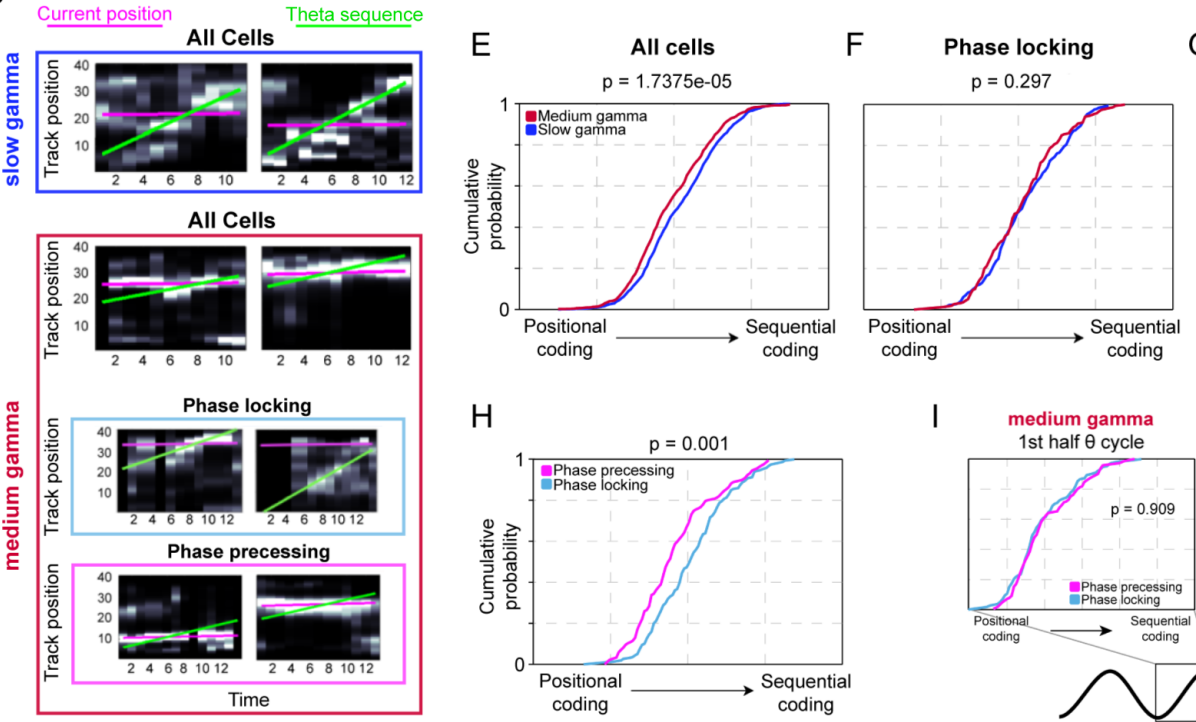

G Phase precessing

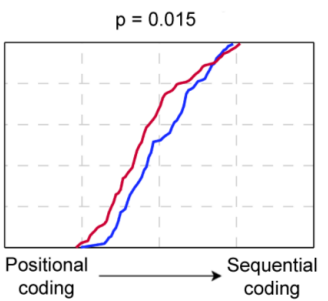

H

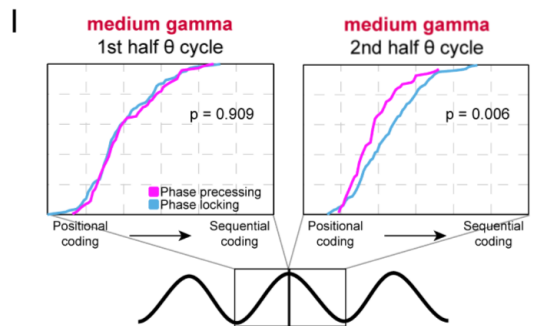

Figure 6: Theta sequences organize during slow gamma dominated network states. (A) Distribution of place field and phase distances for pairs of phase locking or phase precessing cells (Pairwise Measure). (B) Place field distance vs phase distance regression for different gamma coefficients and populations (regression lines were aligned to their first bin on the x-axis). Same regression lines shown in panel (A). (C) Summary of regression slopes of panel (B). (D) Examples of encoded position (y-axis) reconstruction over an entire theta cycle (x-axis), dividing theta cycles according to the corresponding slow-medium gamma power balance and isolating spikes from specific groups of cells (phase locking and phase precessing). Grey color scale: posterior probability from Bayesian Decoding. Violet line: real position of the animal, used to compute the amount of local coding as the probability density around the line. Green line: best positive-slope fit of probability distribution, used as an estimation of sequential non-local cell activation. (E) Sequential coding is more present during periods of high slow/medium gamma power ratio ( $\mathrm{p}<0.01$; Two-sample Kolmogorov-Smirnov test). (F) When reconstructing temporal sequences using the phase locking cells, sequential coding is found during both slow and medium gamma dominated periods ( $\mathrm{p}>0.05$; Two-sample Kolmogorov-Smirnov test). (G) When reconstructing temporal sequences using the phase precessing cells, sequential coding is more frequent during slow gamma dominated periods $(\mathrm{p}<0.05$; Two-sample Kolmogorov-Smirnov test). (H) Sequential coding is more frequent when reconstructing temporal sequences using phase locking cells only, compared to using phase precessing cells only ( $\mathrm{p}<0.01$; Two-sample Kolmogorov-Smirnov test). (I) In the first half of medium gamma dominated theta cycles, phase precessing and phase locking both encode a similar amount of sequential information ( $\mathrm{p}>0.05$; Two-sample Kolmogorov-Smirnov test). In the second half of medium gamma dominated theta cycles, sequential coding is more frequent when reconstructing temporal sequences using phase locking cells only $(\mathrm{p}<0.01$; Twosample Kolmogorov-Smirnov test). 


\section{Discussion}

The data we presented here highlights the variability and heterogeneity of temporal organization modes in CA1 activity. Temporal coding at the single cell (phase precession) and population level (theta sequences), emerge independently, and in association with two different network states marked by strong medium and slow gamma respectively.

We first showed that phase precession is not a feature of all place cells (Figure 2). Our Theta Score measure delineates a continuum between purely phase locked and phase precessing cells, with no or very little correlation with spatial tuning strength, place field size and other basic parameters. Cells at the "precessing" and "locked" ends of this spectrum differ in non space-related properties, such as their theta phase locking during active behavior (or during REM sleep; Figure S9, suggesting that intrinsic differences in anatomy and physiology, as for example belonging to the deep or superficial pyramidal sublayer (Mizuseki et al., 2011, Valero et al., 2015, Valero and de la Prida, 2018, Sharif et al., 2021) may affect to some extent a place cell's propensity to phase precess. Yet, by analyzing phase precession as a function of location on the track (Figure 3) we show that the same place cell may vary substantially its phase precessing behavior at different locations on the track. Thus, intrinsic cell properties may explain a small portion of the total variance at best, and the causes of phase precession may have to be searched not only in the circuit layout, but, most poignantly, in the precise constellation of inputs, cellular and network states affecting a neuron in a given contingency.

An important index of the CA1 network state is the instantaneous intensity of gamma oscillations in multiple frequency ranges. It has been suggested that slow and medium gamma oscillations are proxies for the activation of different interneuronal circuits, and communication with, respectively, CA3 and the Entorhinal Cortex (Bragin et al., 1995, Colgin et al., 2009, Schomburg et al., 2014, Fernández-Ruiz et al., 2017). Interneuron circuits in CA1, including oriens-lacunosum moleculare (O-LM) and bistratified stratum radiatum interneurons may dynamically shift the balance between the two inputs. (Leão et al., 2012, Tukker et al., 2013, Klausberger and Somogyi, 2008, Navas-Olive et al., 2020). We characterized CA1 gamma oscillation by making use of a new device Guardamagna et al. 2021) (Figure 1), combining high-volume, reliable and durable ensemble recording with tetrodes and laminarly resolved LFP with a silicon probe. The LFP is laminarly resolved and measured with a separate set of recording sites from those used to record single units, avoiding any spillovers of spiking activity in the LFP, which may bias LFP-spikes association measures (Buzsáki et al., 2012, Lasztóczi and Klausberger, 2016). We then used a novel analytical procedure (Figure 4), derived from Lasztóczi and Klausberger (2014) in order to separate the different generators. Importantly, this procedure makes explicit use of preferred theta phase information, which effectively distinguishes between generators. Compared to Independent Component Analysis, that has been sometimes used to the same purpose (Schomburg et al., 2014, Fernández-Ruiz et al. 2017) this procedure does not downplay existing correlations between the generators, that may arise for example because of similar or opposite theta phase preferences.

With this precise assessment of the network state, we could show how phase precession only arises in theta cycles where medium gamma was the dominating oscillation. In cycles dominated by slow gamma, even phase 
precessing fields displayed a phase locked firing profile, with spikes concentrating in the ascending phase of the slm theta. With increasing medium gamma, a new cluster of spikes emerges in the position by firing phase plane (Figures 5 and 7), at a further position in the field and at the trough of the slm theta. It is the shift in phase and location between these two clusters - that were first described by Yamaguchi et al. (2002) - that induces the circular-linear correlation between firing phase and location, and therefore the phase precession effect. This pattern is consistent with a number of observations in the literature. For example, the fact that slow gamma induces a backward (prospective coding (Battaglia et al., 2004)) shift in place field location (Bieri et al., 2014) whereas the field moves forward (retrospective coding) with elevated medium gamma. We reproduced this effect in our data (Figure S11 A-B). Furthermore, spike-triggered averages of medium and slow gamma power respectively increase and decrease with place field traversal (Lasztóczi and Klausberger, 2014) (also reproduced here Figure S12, but see (Fernández-Ruiz et al. 2017)).

Interestingly, this exact pattern of theta-phase and spatial offsets was assumed in a theoretical model of phase precession by (Chance, 2012). In this model - as already hypothesized by Yamaguchi et al. (2002) - the cluster corresponding to entry into the place field and later phase is ascribed to CA3 inputs (Figure 7). EC3 inputs would be instead responsible for the spike cluster at the exit of the place field. The theta phases of the two clusters is consistent with CA3 inputs lagging EC3 inputs by a quarter theta cycle (Mizuseki et al., 2009, Montgomery et al., 2009), and with a link between medium and slow gamma and inputs from, respectively, EC3 and CA3 into CA1 (Colgin et al., 2009, Belluscio et al., 2012, Carr et al., 2012, Kemere et al., 2013, Bieri et al., 2014, Schomburg et al. 2014, Lasztóczi and Klausberger, 2014). Crucially, we show here that while the "CA3" spike cluster is always present, the "EC3" cluster appears dynamically only when medium gamma is strong. Thus, there appears to be no stable phase coding for space. Firing theta phase is instead determined by the precise state of CA1 inputs, carrying potentially much richer information. Indeed, the key role of EC3 inputs in supporting phase precession has been underlined by the finding that Medial Entorhinal Cortex lesions abolish precession while leaving place-related activity intact (Schlesiger et al. 2015).

The role of the CA1 interneuronal networks, with its diversity of theta phase locking patterns (Klausberger and Somogyi, 2008) is likely to be key here. In particular, O-LM interneurons can inhibit distal apical dendrites of CA1 pyramidal cells, where EC3 afferents terminate, and can inhibit interneurons targeting the stratum radiatum (receiving CA3 inputs). Thus, with a inhibition/disinhibition mechanism, O-LM cells can shift the balance between the two inputs streams (Leão et al. 2012). Together, our data suggest that the two-spike cluster structure of the Chance (2012) model is created by the interplay of two distinct dynamical states, whose modulation in time determines the appearance or disappearance of the phase precession pattern. Last, we found evidence for a decoupling of phase precession and theta sequences. Phase locked place cells expressed sequential activity most reliably. Phase precessing cells only expressed sequences during periods of strong slow gamma, that is, when phase precession was absent (Figure 6). Furthermore, theta sequences were concentrated in a theta phase range that corresponds to the "CA3" related portion of the precession cycle (Yamaguchi et al., 2002. Chance, 2012). Interpreting these results in the theoretical framework delineated above, we may say that spikes emitted earlier in the theta cycle, when EC3 inputs are strong, tend not to merge into sequences and may 
bioRxiv preprint doi: https://doi.org/10.1101/2021.12.22.473863; this version posted December 23, 2021. The copyright holder for this

A

Slow Gamma dominates

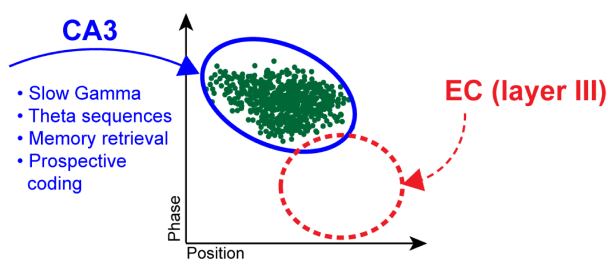

Medium Gamma dominates

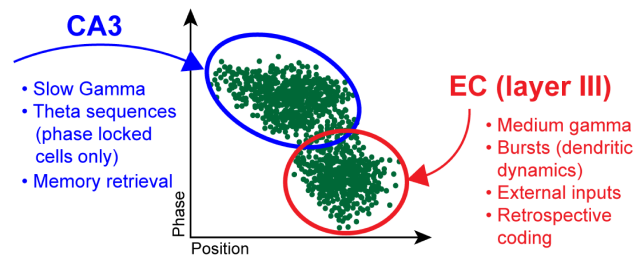

B

Slow Gamma dominates

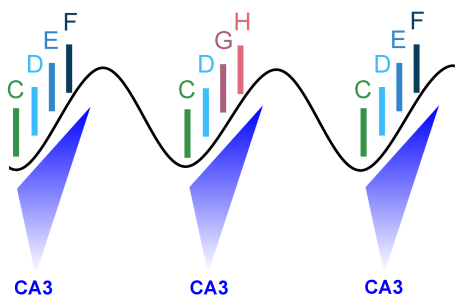

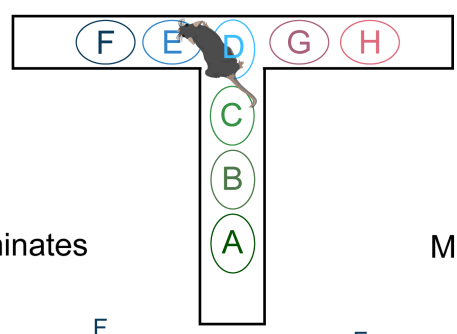

Medium Gamma dominates

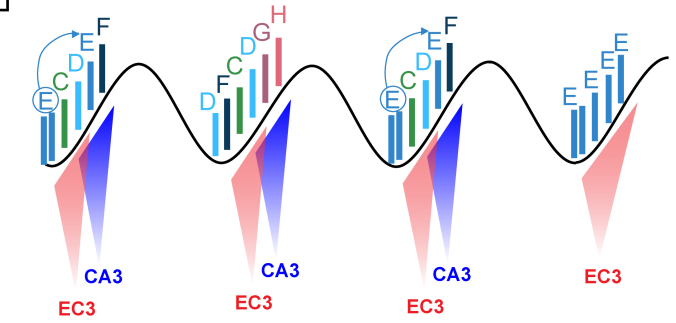

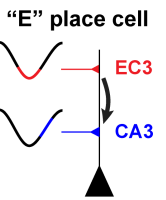

Figure 7: The "dual network states" model of temporal coding in the hippocampus. Our observations, and those of others in the literature, can be summarized by a phenomenological model assuming that the CA1 network spontaneously fluctuates between two dynamical states. (A) Emergence of the phase precession pattern. When slow gamma is dominant (left), the firing of place precessing place cells is restricted to the portion of the theta cycle coincident with CA3 inputs. Spiking takes place in the initial portion of the place field, so that spatial tuning is prospective. In the position-phase plane, spikes group in the cluster highlighted by the blue contour. Both phase locked and phase precessing cells display theta sequences in this state, that may be conducive to retrieving information stored in CA3's memory networks. Because of the clustering of spikes, no phase precession effect is visible. When medium gamma is present (right), a second cluster of phase precessing cells spikes appears on the position-phase plane, shifted towards an earlier theta phase, corresponding to EC3 activation and to a further position in the place field, here highlighted by the red contour. The relative position of the two clusters gives rise to the phase precession effect (Chance, 2012). Bursts are most prominent in the second cluster, suggesting the influence of dendritic dynamics triggered by EC3 afferents depolarizing distal apical dendrites of pyramidal cells. The interference between spikes in the two clusters disrupts theta sequences, which are still apparent in phase locked phase cells. This state may favor the combination of memory-derived and new sensory information. (B) Consequences of this hypothesis for theta sequences. Examples of possible spike timings during theta cycle when slow gamma (left) or medium gamma (right) dominate. Inset at the bottom schematizes the role of plateau potentials in plasticity in our model. Illustration of the mouse adapted from SciDraw (doi.org/10.5281/zenodo.3925997). 
even be considered a "disturbance" to time-ordered activation of cell assemblies (Figure 7). Phase precession has been widely attributed the role of shaping hippocampal ensembles activity in compressed theta sequences. The time lag between successive spikes of two cells in the sequence by this mechanism would be brought to a range that is compatible with spike-timing dependent plasticity mechanisms (Skaggs and McNaughton, 1996 Reifenstein et al. 2021). Our results call for a revision of this theoretical stance. That phase precession and theta sequences can occur independently has been shown by analyzing single lap ensemble activity (Feng et al. 2015). Also Middleton and McHugh (2016) showed that genetically disconnecting CA3 from CA1 selectively disrupts theta sequences but not phase precession, and the same patterns was observed when decoupling locomotor activity from movement in space (Drieu et al. 2018). Here, we go beyond that by showing how the dynamical determinants of these two properties of CA1 activity are distinct, which casts doubts on precession as the key mechanism for sequence encoding. Other factors likely shape synaptic plasticity and favor sequence emergence. For example, that slow gamma is conducive to stronger sequential activity is interesting, because during sleep sharp-wave ripples, the same oscillation is related to stronger replay of sequences (Carr et al., 2012). It is possible that CA3 inputs, representing retrieval of sequences from memory, and the gamma-modulated firing of radiatum-targeting bistratified interneurons (Tukker et al., 2013) may contribute to this enhancement of temporal ordering.

Yet, how the gap between the time scale of behavior (hundreds of milliseconds to seconds) and the time scale of synaptic plasticity (milliseconds) may be bridged remains to be seen. One possibility is suggested by recent data on dendritic dynamics, showing that "plateau potentials" induced by EC3 inputs on distal apical dendrites may last for hundreds of milliseconds (Bittner et al., 2015, 2017) and may enable linking of inputs occurring within that time interval (Milstein et al. 2021). We may speculate that the EC3 inputs act as an enabling signal, favoring the potentiation of synapses from CA3 with matching spatial tuning Remondes and Schuman, 2002). Such a mechanism could explain how a coherent spatial representation merging information retrieved from memory in CA3 and novel environmental information may be formed, and how sequences may be updated and refined. In Figure $7 \mathrm{~B}$ we formulate an initial hypothesis for this. In our model, when slow gamma dominates (left panel) activity reflects predictive, sequential activations driven by the CA3 input. Activity will "look ahead" of the current position (also spanning potential alternative paths (Johnson and Redish, 2007)). During periods of medium gamma of medium gamma prevalence (right panel) activity will reflect a combination of CA3 inputs (predictive, sequential) and EC3 input (tuned on current position, place cell "E" in the diagram). When EC3 input is prevalent (rightmost case) only positional code is observed, matching our observations in Figure 6. When the two inputs are roughly balanced (first three cases), the EC3 driven spikes will disrupt theta sequences, so that no orderly activation will be observed. We speculate however, that EC3 inputs taking place at the slm theta trough trigger plateau potentials at distal apical dendrites, which may "tag" the dendritic tree so that they may facilitate synaptic plasticity at stratum radiatum synapses when the same cells is activated by the CA3 inputs, reinforcing output for validated predictions. The duration of the plateau potentials easily spans this theta phase range (Bittner et al. 2015).

A hint that dendritic dynamics may play a role is the fact that the theta phase preference of spike bursts 
(which depend on dendritic potentials lasting for several milliseconds (Bittner et al., 2015, Milstein et al., 2021)) is different from phase preference for single spikes and peaks in the phase range of the "EC3" spike cluster (Figure 5F) (see also (Mizuseki et al., 2009)).

In conclusion, we showed here how two different dynamical states support respectively phase precession and theta sequences. These two states link to strong medium and slow gamma and may be associated to the influence of EC3 and CA3 on CA1. We argue that these two states may actually correspond to different computational operations, with the slow gamma state supporting memory based predictions (e.g. of future animal position) and medium gamma fostering influx of new information from the external world. CA1 functional role within the hippocampal circuit might reside in its ability to dynamically fluctuate between these two modes of operation based on cognitive demands on the task (Takahashi et al. 2014), on a temporary bias between cognitive modes (Cabral et al. 2014) or even spontaneously in the absence of cognitive demands, like in the data presented here. The heterogeneity of phase-dependent activity across the population makes sure that the output of CA1 effectively "multiplexes" these two streams, possibly accommodating time-varying computational demands in other brain structures. CA1 would also play a key role for learning, by comparing and merging internally generated (e.g. from memories stored in CA3) predictions and current sensory information, that may both act as a "supervisor" signal for prediction validation and integrate the retrieved memory with information richer in detail (Treves and Rolls, 1994). We hypothesize that CA1's specific role in the hippocampal circuitry is the flexible and dynamical combination of these two modes.

\section{Materials and Methods}

\section{Ethics statement}

In compliance with Dutch law and institutional regulations, all animal procedures were approved by the Central Commissie Dierproeven (CCD) and conducted in accordance with the Experiments on Animals Act (project number 2016-014 and protocol numbers 0029).

\section{Animals}

6 male C57BL6/J mice (Charles River) were used in this study, all implanted with a Hybrid Drive. All animals received the implant between 12 and 16 weeks of age. After surgical implantation, mice were individually housed on a 12-h light-dark cycle and tested during the light period. Water and food were available ad libitum.

\section{Surgical Procedures}

The fabrication of the Hybrid Drives and the implantation surgeries were done as described earlier Guardamagna et al., 2021). Specifically, mice were anaesthetized with $1.25 \%-2 \%$ isoflurane and injected with analgesia (Carprofen, $5 \mathrm{mg} / \mathrm{kg} \mathrm{SC}$ ). Then, mice were placed in a stereotaxic frame and antibacterial ophthalmic ointment (Alcon, UK) was applied to the eyes. A circular piece of dorsal scalp was removed and the underlying skull was 
cleaned and dried. 3 stainless steel M0.8 screws were used to secure the drive (1 ground screw in the frontal plate, screw in the parietal plate opposite to micro-drive, 1 screw in the occipital plate). A craniotomy was made over the right cortex (top-left corner at AP: $-1.20 \mathrm{~mm}$; ML: $0.6 \mathrm{~mm}$ relative to bregma; bottom-right corner at AP: $-2.30 \mathrm{~mm}$; ML: $2.10 \mathrm{~mm}$ relative to bregma) using a 0.9 Burr drill. The dura was removed and the array of the drive was slowly lowered into the brain with the silicon probe shaft already adjusted at the final depth. The guide tube array was lowered right above the brain surface and the craniomoty was filled with sterile vaseline to protect the brain and the array from cement flowing in. The drive was cemented onto the skull using dental adhesive (Superbond C\&B, Sun Medical, Japan) and tetrodes were individually lowered into the brain ( 5 turns - $\approx 900 \mu \mathrm{m}$ ) using the screw-spring mechanism. Mice were allowed to recover from surgery for at least seven days before experiments began.

\section{Neural and behavioral data collection}

From post-surgery day 3 onward, animals were brought to the recording room and electrophysiological signals were investigated during a rest session in the home cage. Tetrodes were lowered individually, in $45 / 60 \mu \mathrm{m}$ steps, until common physiological markers for the hippocampus were discernible (SWR complexes during sleep or theta during locomotion). The majority of the tetrodes reached the target location (CA1 pyramidal layer) in 7-10 days. Silicon probe signals were used as additional depth markers.

Electrophysiological data were recorded with an Open Ephys acquisition board (Siegle et al., 2017). Signals were referenced to ground, filtered between 1 and $7500 \mathrm{~Hz}$, multiplexed, and digitized at $30 \mathrm{kHz}$ on the headstages (RHD2132, Intan Technologies, USA). Digital signals were transmitted over two custom 12wire cables (CZ 1187, Cooner Wire, USA) that were counter-balanced with a custom system of pulleys and weights. Waveform extraction and automatic clustering were performed using Dataman (https://github. com/wonkoderverstaendige/dataman) and Klustakwik (Harris et al., 2000), respectively. Clustered units were verified manually using the MClust toolbox. During all experiments, video data was recorded using a CMOS video camera (Flea3 FL3-U3-13S2C-CS, Point Grey Research, Canada; 30 Hz frame rate) mounted above the linear track.

\section{Behavioral paradigm}

Each behavioral session was preceded and followed by a rest session in the animal's home cage ("Pre sleep" and "Post sleep") that typically lasted between 60 and 120 minutes each. For the behavioral paradigm, mice were positioned at one end of a 1-meter long track with the task to run to the other end to collect a reward (a piece of Weetos chocolate cereal). After the animal consumed the reward, another reward was positioned on the opposite end. A lap was defined as an end-to-end run where the animal's body started from the first $10 \mathrm{~cm}$ of the track and reached the last $10 \mathrm{~cm}$ at the other end of the track without returning to its initial position. Recordings typically lasted between 20 and 30 minutes and experiments were performed on 10 consecutive days. Mice were never food or water deprived. 


\section{Histology}

After the final recording day tetrodes were not moved. Animals were administered an overdose of pentobarbital $(300 \mathrm{mg} / \mathrm{ml})$ before being transcardially perfused with $0.9 \%$ saline, followed by $4 \%$ paraformaldehyde solution. Brains were extracted and stored in $4 \%$ paraformaldehyde for 24 hours. Then, brains were transferred into $30 \%$ sucrose solution until sinking. Finally, brains were quickly frozen, cut into coronal sections with a cryostat (30 $\mu \mathrm{m})$, mounted on glass slides and stained with cresyl violet. The location of the tetrode tips was confirmed from stained sections (Figure S1), in combination with previously mentioned electrophysiological markers.

\section{Neural data analysis}

Before applying other analysis, continuous (LFP) signals were down-sampled to $1 \mathrm{kHz}$. Most importantly, LFP across the 16 contacts was used to compute the corresponding Current Source Density signal (Buzsáki et al. 2012). Since this transformation is based on computing the discrete laplacian along the probe axis (that is along the direction running through CA1 layers), the resulting CSD signal is limited to 14 channels (16 minus the 2 extremes).

\section{Basins}

The CSD signal was used to compute the phase-amplitude coupling strength between a reference theta oscillation and faster oscillations comprised in a $[15250] \mathrm{Hz}$ range. We first applied a wavelet transform to the CSD to obtain the analytical signal over time and across a broad each of these regions, a basin. Small Basins (comprising less than 40 bins in total) were removed from visualizations to improve readability. The same data was then used to track the evolution of the phase-amplitude couplings across different layers and frequencies. For each of the identified main gamma frequency ranges (slow [20-45] Hz; medium [60-90] Hz; fast [120-180]Hz) we extracted layer-specific coupling strength and phad range of frequencies. We then separately obtained the instantaneous phase of the theta oscillation (taken between $6 \mathrm{~Hz}$ and $10 \mathrm{~Hz}$ ) and the amplitude at the higher frequencies. Theta oscillation reference was generally taken from the stratum lacunosum moleculare, where theta modulation of the LFP is stronger, but the same procedure using a theta from another layer gave equivalent results. Instantaneous theta phase was binned (32 bins) and for each interval the average amplitude of oscillations at higher frequency was computed. We then applied a discrete laplace operator to the frequency-phase matrix so obtained, to identify portions of the matrix characterized by negative curvature. These regions are found by requiring the laplace operator to be negative. For each of these regions, we further selected only the bins that showed a coupling strength above 1STD of the mean of the coupling of the respective frequency. Such operation was repeated for the CSD computed for each contact of the probe. After this double thresholding, each of the layer-specific coupling matrices was then reduced to a sparse version in which only significantly coupled regions had non-zero values. To identify continuities and changes in the couplings across hippocampal layers we then stacked all the sparse-coupling matrices on top of each other, adding a 3rd dimension to the coupling arrangement. Again, we looked for continuous 3D regions of significant bins. We dubbese of the maximum coupling. In the rest of 
the paper slow gamma power was computed using the signal extracted from the radiatum layer, while medium gamma power was taken from the probe contact in the stratum lacunosum moleculare.

\section{REM Sleep Detection}

Following an established practice (Mizuseki et al., 2009, 2011), periods of REM sleep were classified offline based on the ratio between LFP power in the theta frequency range $(5-9 \mathrm{~Hz})$ and the power in delta range $(2-4 \mathrm{~Hz})$. REM periods were identified in the rest period that followed exploration sessions by combining the theta/delta ratio with an additional requirement for absence of animal movement.

\section{Gamma Coefficient}

The instantaneous balance between the power in the slow gamma $\left(P_{\text {Slow }}(t)\right)$ and medium gamma $\left(P_{M e d}(t)\right)$ frequency range was computed as follow. First both the slow gamma power and medium gamma power during running periods were separately z-scored. Then a power-ratio score, spanning the [-1 1$]$ interval was computed as:

$$
\gamma=\frac{P_{\text {Slow }}^{z}(t)-P_{M e d}^{z}(t)}{P_{S l o w}^{z}(t)-P_{M e d}^{z}(t)}
$$

so that a value of -1 would correspond to total medium gamma domination and +1 to slow gamma completely dominating.

\section{Place Cell Identification}

Putative excitatory pyramidal cells were discriminated using their auto-correlograms, firing rates and waveform information. Specifically, pyramidal cells were classified as such if they had a mean firing rate $<8 \mathrm{~Hz}$ and the average first moment of the autocorrelogram (i.e., the mean value) occurring before 8 milliseconds (Csicsvari et al. 1999). Only cells classified as pyramidal cells were used for further place cell analysis. Place cells were defined applying a combination of different criteria. All analysis were performed on speed-filtered activity, after removing periods in which the animal speed was smaller than $5 \mathrm{~cm} / \mathrm{s}$. Then, only cells with an average activity above $0.3 \mathrm{~Hz}$ were taken into account. Then for each of these cells, the Skaggs information per second $I=\sum_{x} \xi(x) \log \frac{\xi(x)}{\langle\xi\rangle_{x}}$ was compared to distribution of information values resulting from randomly shuffling cell spike times. A cell passed the selection if its Skaggs information was significantly higher than the ones of the surrogate distribution. Last, only place cells with peak firing rate higher than $1 \mathrm{~Hz}$ were kept for further analysis. Place fields were isolated as continuous regions in the rate map with rate higher than $20 \%$ of the cell maximum activity. Multiple fields (field one and field two) were then sorted according to their respective peak firing rate.

The modulation of place field shape by gamma power was evaluated by first estimating the place field boundaries using all spikes from a cell. Then the place field extension was divided in 7 spatial bins. For each of these bins we computed the spike rate of the cell using only spikes emitted in association of a specific range of the slow-to-gamma power ratio. For each cell the overall spike probability over the bins was normalized to 
1 and the final value for each bin was obtained as an average over all cells in the dataset. Opposite running directions were treated separately.

\section{Place Cell Classification}

Spikes were filtered both in speed $(>5 \mathrm{~cm} / \mathrm{s})$ and in position, by taking only those emitted within the boundaries of the cell place field (field one in the case of multiple fields). For each place cell, we measured to what degree the phase (with respect to theta oscillations) of spikes was concentrated and to what extent it was modulated by the respective position within the place field. In the first case we computed the length of the Reyleigh vector associated to the distribution of spike phases with respect to the instantaneous theta phase (slm theta). In the second case we used a circular-linear correlation to measure the degree of dependency between the spike phase and the position within the field. Both measure yielded a score between 0 and 1, with 1 meaning perfect phase locking and perfect phase-position relation, respectively. We combined the two scores into a 'Theta Score' subtracting one from the other: Theta Score = Precession Score - Locking Score. Because of the similar interval of the two measures, negative Theta Scores would indicate spikes probability being mostly modulated by specific phases of theta, while positive Theta Scores would point to a substantial presence of phase precession.

\section{Single Spikes and Burst Spikes}

We subdivided spikes from a cell into single and bursts by applying a temporal interval filter. Any spike occurring within $7 \mathrm{~ms}$ of another was labelled as belonging to a burst, all the others were considered as single spikes. If not otherwise stated, spike probability evaluation and other analysis were performed using only single spikes. Burst spikes were analyzed separately.

Significance of theta phase selectivity for either single or burst spikes was evaluated with a shuffling procedure. The probability of spikes in any bin along the $\left[\begin{array}{ll}0 & 2 \pi\end{array}\right]$ interval was computed by dividing the number of spikes of each cell by the probability of observing a specific gamma coefficient in the same theta phase interval. For each cell, this ratio was compared to an equivalent measure applied to a surrogate version of the cell activity, where spike times had been randomly reassigned to any of the temporal bins characterized by a slow-to-medium gamma power ratio in a specific range. Such comparison was repeated 1000 times for each cell, each time repeating the shuffling procedure. Statistics were computed on the distribution of differences between the real spike distribution and the surrogate ones.

\section{Generalized-Linear Model (GLM)}

As described in previous work (Dunn et al. 2015) we used a maximum entropy model inference paradigm to reconstruct the distribution of each cell's firing probability. As a statistical model, we considered the maximum entropy model known as kinetic Ising model. We first separated running periods from periods of quiescence by applying a $5 \mathrm{~cm} / \mathrm{s}$ speed filter. The activity of the cells was binned in $10 \mathrm{~ms}$ bins, and a binary variable $S_{i}(t)$ was assigned to each neuron for each temporal bin. $S_{i}(t)$ was taken to be a binary variable, with $+1 /-1$ values, 
depending on the presence/absence of spikes emitted by neuron i within time bin t. Since the length of time bins was relatively short, this was a reasonable approximation as the case of multiple spikes per bin was rare. Letting the state of each neuron at time $t$ depend on the state of the population in the previous time step $t-$ 1 , the maximum entropy distribution over the state $S_{i}(t)$ of neuron i at time $\mathrm{t}$ is

$$
P\left(S_{i}(t)\right)=\frac{\exp \left[S_{i}(t) H(t-1)\right]}{2 \cosh [H(t-1)]}
$$

where $H(t)$ is a time dependant covariate having the role of the external field in statistical physics. Eq. 1 defines a GLM (Generalized Linear Model), where, in each time bin, mostly only one or zero spikes per bin are observed and the interaction kernel extends one time step in the past. To find what values of $H(t)$ are the most likely to generate the observed data given Eq. 1, we maximized the log-likelihood function

$$
\mathcal{L}[S, H]=\sum_{i, t} S_{i}(t) H_{i}(t-1)-\log \left[2 \cosh \left[H_{i}(t-1)\right]\right]
$$

with respect to $H(t)$. The log-likelihood measures how well the model explains the statistics in the observed data. In our analysis, we have used the natural logarithm. Since the external field, $H(t)$, can explain the variations in the firing rate as the rat navigates in space, it becomes important to model it appropriately. Here we assumed that the spatial input arises as the sum of one-dimensional Gaussian basis functions centered on evenly spaced L intervals covering the linear track. Opposite running directions were treated as distinct environments and analyzed separately. The spatial field of cell $i$ at time $t$ is then

$$
H_{i}^{\text {Spat }}(t)=\sum_{k} \alpha_{i k} \exp \left[-\left(x-x_{k}\right)^{2} / l^{2}\right]+h_{i}
$$

where $h_{i}$ is a unit-specific spatially and temporally constant baseline, and $x_{k}$ and $l$ are the centers of the regular intervals and the widths of the basis functions, respectively. We expanded this purely spatial description of cell activity by combining the spatial contribution to activity with that of specific phases of theta oscillation and a specific slow-medium gamma balance. To do so, we tiled the 3-dimensional space obtained from combining i) the position on the track, ii) the theta phase (in the $\left[\begin{array}{ll}0 & 2 \pi\end{array}\right]$ interval) and the relative strength of the instantaneous power in the slow and medium gamma range, computed as a normalized difference: $\gamma=\frac{P_{S l o w}^{z}(t)-P_{M \text { Med }}^{z}(t)}{P_{\text {Slow }}^{z}(t)-P_{\text {Med }}^{z}(t)}$ (spanning the [-1 1 1] interval), where the $z$ apex indicates previous z-scoring of both power time-series. Such space was tiled with a regular square lattice of $\mathrm{L} \times \mathrm{T} \times \mathrm{G}$ three-dimensional Gaussians, with 0 off-diagonal terms and variance $l, t$ and $g$ in the three directions. The external field of cell $i$ can thus be expressed as:

$$
H_{i}(t)=\sum_{k q p} \alpha_{i k q p} \exp \left[-\left(x-x_{k}\right)^{2} / l^{2}-\left(\phi-\phi_{q}\right)^{2} / t^{2}-\left(\gamma-\gamma_{p}\right)^{2} / g^{2}\right]+h_{i}
$$

where we introduced the dependency on the current phase $\phi$ of the theta oscillation and current gamma balance $\gamma$. An accurate representation of the cell activity in the space-phase configuration, can be found by inferring the parameters $\alpha_{i k q p}$ of the linear combination of the Gaussian basis function. We first optimized the 
values of $\mathrm{L}$ and $\mathrm{T}$ (the number of Gaussian basis functions in the lattice) and l, $\mathrm{t}$ (their widths), while keeping $\mathrm{G}$ and $\mathrm{g}$ fixed $(\mathrm{G}=6$ and $\mathrm{g}=0.4)$. We maximized the likelihood over a range of values of $\mathrm{L}$ (from 15 to 25$)$, $\mathrm{T}$ (from 4 to 10 ), 1 (from 5 to $30 \mathrm{~cm}$ ) and $\mathrm{t}$ (from 0 to $\pi / 2$ ) and chose the values of the parameters that gave the highest Akaike-adjusted likelihood value. The Akaike information criterion (AIC) is a measure to compensate for overfitting by models with more parameters, where the preferred model is that with the minimum AIC value, defined as

$$
A I C=2 \ln \left(\mathcal{L}\left[S, H,(L, T, l, t)_{M L}\right]\right)+2 n
$$

where $L$ is the likelihood at the maximum likelihood (ML) estimates of the parameters $\alpha$ 's and $h_{i}$ ) for a given value of $\mathrm{M}, \mathrm{N}, \mathrm{r}$ and $\mathrm{p} . \quad n$ is the number of parameters (here $\mathrm{r}$ and $\mathrm{p}$ do not affect this number as it is a scale factor for Gaussian basis functions, while larger values of $\mathrm{M}$ and $\mathrm{N}$ result in more parameters $\alpha$ included in the model). The procedure was performed over all available sessions at once so that the resulting optimal parameters $(\mathrm{L}=20, \mathrm{~T}=6$ and $\mathrm{l}=8 \mathrm{~cm}, \mathrm{p}=\pi / 3)$ were applied to all of them. Firing rate maps can then be expressed for an arbitrary combination of position, phase and gamma balance as

$$
H_{i}(t)=\sum_{k q p} \alpha_{i k q p} \exp \left[-\left(x-x_{k}\right)^{2} / l^{2}-\left(\phi-\phi_{q}\right)^{2} / t^{2}+-\left(\gamma-\gamma_{q}\right)^{2} / g^{2}\right]+h_{i}
$$

where $x, \phi$ and $\gamma$ are the desired values of position, theta phase and gamma balance. In all our analysis, we considered a partition of the environment in bins of $2.5 \mathrm{~cm}$. The effects of speed on the firing probabilities was addressed in a similar manner, by including the instantaneous velocity of the animal as a further covariate in the Gaussian decomposition method. The GLM was then ran to infer a set of $\alpha_{i k q p z}$ weights covering a 4D space of position $\mathrm{x}$ phase $\mathrm{x}$ gamma $\mathrm{x}$ speed. The parameters of the Gaussian-basis along the speed dimension were not optimized but were taken as $V=4$ Gaussians with a standard deviation of $6 \mathrm{~cm} / \mathrm{s}$.

\section{Pairwise Spike Timing}

For each cell, spikes emitted during running were further filtered so that: i) only first spikes in each theta cycle (taking the peak of the theta oscillation as the starting point of the cycle) and ii) only spikes emitted within the cell place field one, were considered. For each of these spikes we also computed the simultaneous slow $/$ medium gamma power ratio which we then used as a label to further subdivide the spikes. We then considered all the cell pairs in the population of simultaneously recorded place cells. Each pair (A,B) of place cells was then defined by i) the spatial distance between the place fields of cell A and B (computed as the distance between the centers of mass, but using the distance between the fields peaks did not change the results) and ii) the average phase interval between the spikes of the two cells. The latter was computed by taking spikes of cell A as reference and within each theta cycle (for which a spike from both cells was available) taking the $d \theta_{A->B}(k)$ (where $\mathrm{k}$ stands for the kth theta cycle), that is the (signed) difference between the phase of the spike of cell $\mathrm{A}$ and that of cell B spike. We then computed the center of mass of the $d \theta_{A->B}$ distribution and used it as a measure of the average phase offset between that cell pair. Spatial distance and phase distance were then arranged in a 
2-dimensional space and the average phase offset was computed over different ranges of spatial distances. The analysis was repeated using cell pairs from specific populations subgroups and by restricting the use of spikes to those emitted in the presence of a certain gamma balance.

\section{Bayesian decoding}

To reconstruct the position on the track encoded in theta associated spiking activity, we implemented a standard Bayesian decoding procedure (Zhang et al., 1998). Periods of active locomotion were first segmented using the peaks of theta oscillation (extracted from the stratum lacunosum moleculare). Each theta period was further subdivided using a sliding window of $30 \mathrm{~ms}$ length and with an offset of $10 \mathrm{~ms}$. A population vector was then built for each of these sub-windows $\boldsymbol{\eta}(t)$, where $\mathrm{t}$ indicates the time within the theta cycle subdivision. The likelihood $P(x \mid \boldsymbol{\eta}(t))$ of position on the track $x$ given the population activity was then computed according to Bayes' formula using the set of probabilities $P(\boldsymbol{\eta}(t)) \mid x$ ) obtained from marginalizing the result of the GLM (see above) over the phase of spiking. The resulting probability density $P(x, t)$ was normalized so that $\sum_{x} P(x, t)=1$ for each time window $t$ that contained 3 spikes or more, and was otherwise set to 0 and ignored in the following analysis. Using the normalized probability density, for each theta cycle we computed two scores. We first evaluated the amount of 'local' information in the activity by summing the probability concentrated around the current position of the animal $\Upsilon_{\text {Local }}=\sum_{t} P\left(x^{*}, t\right)$ where $x^{*}$ denotes the spatial bin corresponding to the animal position at the time $t$. The presence of 'non-local' sequence-like activity spanning a significant spatial interval around the animal position was instead evaluated by running a set of linear regression over the $P(x, t)$ matrix. Among these regressions, we searched for the best-fit, overlapping with the largest amount of probability density. That is, $\Upsilon_{\text {NonLocal }}=\max _{\beta} \sum_{k} P\left(x_{\beta(k)}, t_{\beta(k)}\right)$ where $\beta$ indicates a set of linear parametrizations of $x$ and $t: x=\beta_{1}+t \times \beta_{2}$. Importantly $\beta_{2}$ was taken to be always $>0$ so to exclude fits very close to the $\Upsilon_{L o c a l}$ defined above. The same procedure was applied using different set of cells to compute the probability density, so to obtain an estimation of the spatial information carried by specific cell groups (in our case, the entire population and either phase precessing or phase locking cells). Theta cycls were further classified according to the simultaneously expressed gamma power ratio. The average ratio between the power of slow and medium gamma over the interval of the theta oscillation was used as a label for that theta cycle and used to subdivide the set of cycles depending on the relative strength of the slow and medium gamma component.

\section{Acknowledgments}

We thank Freyja Olafsdottir, Cliff Kentros and Alessandro Treves for their comments on the manuscript and the Battaglia lab for helpful discussions and comments at different stages of the project. This work was supported by the European Union's Horizon 2020 research and innovation program (MGate, grant agreement no. 765549; M.G. and F.P.B.), the European Research Council (ERC) Advanced Grant "REPLAY-DMN" (grant agreement no. 833964; F.P.B.), European Union's Horizon 2020 Research and Innovation Programme Grant "BrownianReactivation" (grant agreement no. 840704; F.S.). 


\section{Author contributions}

Conceptualization: M.G., F.S. and F.P.B.; Investigation and Data Curation: M.G.; Methodology and Software: M.G, F.S. and F.P.B.; Resources: F.S. and F.P.B.; Formal Analysis and Visualization, F.S. and M.G.; Writing - Original Draft: M.G., F.S. and F.P.B.; Writing - Review \& Editing: M.G., F.S. and F.P.B.; Supervision and Funding Acquisition: F.S. and F.P.B. 


\section{References}

Battaglia, F. P., Sutherland, G. R., and McNaughton, B. L. (2004). Local Sensory Cues and Place Cell Directionality: Additional Evidence of Prospective Coding in the Hippocampus. Journal of Neuroscience, 24(19):4541-4550. Publisher: Society for Neuroscience Section: Behavioral/Systems/Cognitive.

Belluscio, M. A., Mizuseki, K., Schmidt, R., Kempter, R., and Buzsáki, G. (2012). Cross-Frequency Phase-Phase Coupling between Theta and Gamma Oscillations in the Hippocampus. Journal of Neuroscience, 32(2):423435. Publisher: Society for Neuroscience Section: Articles.

Bieri, K. W., Bobbitt, K. N., and Colgin, L. L. (2014). Slow and Fast Gamma Rhythms Coordinate Different Spatial Coding Modes in Hippocampal Place Cells. Neuron, 82(3):670-681.

Bittner, K. C., Grienberger, C., Vaidya, S. P., Milstein, A. D., Macklin, J. J., Suh, J., Tonegawa, S., and Magee, J. C. (2015). Conjunctive input processing drives feature selectivity in hippocampal CA1 neurons. Nature Neuroscience, 18(8):1133-1142. Bandiera_abtest: a Cg_type: Nature Research Journals Number: 8 Primary_atype: Research Publisher: Nature Publishing Group Subject_term: Dendritic excitability;Hippocampus;Navigation;Synaptic plasticity Subject_term_id: dendriticexcitability;hippocampus;navigation;synaptic-plasticity.

Bittner, K. C., Milstein, A. D., Grienberger, C., Romani, S., and Magee, J. C. (2017). Behavioral time scale synaptic plasticity underlies CA1 place fields. Science, 357(6355):1033-1036. Publisher: American Association for the Advancement of Science.

Bragin, A., Jando, G., Nadasdy, Z., Hetke, J., Wise, K., and Buzsaki, G. (1995). Gamma (40-100 Hz) oscillation in the hippocampus of the behaving rat. Journal of Neuroscience, 15(1):47-60. Publisher: Society for Neuroscience Section: Articles.

Buzsáki, G. (2002). Theta Oscillations in the Hippocampus. Neuron, 33(3):325-340.

Buzsáki, G., Anastassiou, C. A., and Koch, C. (2012). The origin of extracellular fields and currents - EEG, ECoG, LFP and spikes. Nature Reviews Neuroscience, 13(6):407-420. Bandiera_abtest: a Cg_type: Nature Research Journals Number: 6 Primary_atype: Reviews Publisher: Nature Publishing Group Subject_term: Cellular neuroscience;Computational neuroscience;Extracellular signalling molecules;Ion channels;Synaptic transmission Subject_term_id: cellular-neuroscience;computational-neuroscience;extracellular-signallingmolecules;ion-channels;synaptic-transmission.

Buzsáki, G., Buhl, D. L., Harris, K. D., Csicsvari, J., Czéh, B., and Morozov, A. (2003). Hippocampal network patterns of activity in the mouse. Neuroscience, 116(1):201-211.

Buzsáki, G. and Moser, E. (2013). Memory, navigation and theta rhythm in the hippocampal-entorhinal system. Nature neuroscience, 16:130-8. 
Cabral, H. O., Vinck, M., Fouquet, C., Pennartz, C. M. A., Rondi-Reig, L., and Battaglia, F. P. (2014). Oscillatory Dynamics and Place Field Maps Reflect Hippocampal Ensemble Processing of Sequence and Place Memory under NMDA Receptor Control. Neuron, 81(2):402-415.

Carr, M. F., Jadhav, S. P., and Frank, L. M. (2011). Hippocampal replay in the awake state: a potential substrate for memory consolidation and retrieval. Nature Neuroscience, 14(2):147-153. Bandiera_abtest: a Cg_type: Nature Research Journals Number: 2 Primary_atype: Reviews Publisher: Nature Publishing Group Subject_term: Hippocampus Subject_term_id: hippocampus.

Carr, M. F., Karlsson, M. P., and Frank, L. M. (2012). Transient Slow Gamma Synchrony Underlies Hippocampal Memory Replay. Neuron, 75(4):700-713.

Chadwick, A., van Rossum, M. C., and Nolan, M. F. (2015). Independent theta phase coding accounts for CA1 population sequences and enables flexible remapping. eLife, 4:e03542. Publisher: eLife Sciences Publications, Ltd.

Chance, F. S. (2012). Hippocampal Phase Precession from Dual Input Components. Journal of Neuroscience, 32(47):16693-16703. Publisher: Society for Neuroscience Section: Articles.

Colgin, L. L., Denninger, T., Fyhn, M., Hafting, T., Bonnevie, T., Jensen, O., Moser, M.-B., and Moser, E. I. (2009). Frequency of gamma oscillations routes flow of information in the hippocampus. Nature, 462(7271):353-357.

Csicsvari, J., Hirase, H., Czurkó, A., Mamiya, A., and Buzsáki, G. (1999). Oscillatory coupling of hippocampal pyramidal cells and interneurons in the behaving Rat. The Journal of Neuroscience: The Official Journal of the Society for Neuroscience, 19(1):274-287.

Davidson, T. J., Kloosterman, F., and Wilson, M. A. (2009). Hippocampal Replay of Extended Experience. Neuron, 63(4):497-507.

Deshmukh, S. and Knierim, J. (2011). Representation of Non-Spatial and Spatial Information in the Lateral Entorhinal Cortex. Frontiers in Behavioral Neuroscience, 5:69.

Dragoi, G. and Buzsáki, G. (2006). Temporal Encoding of Place Sequences by Hippocampal Cell Assemblies. Neuron, 50(1):145-157.

Drieu, C., Todorova, R., and Zugaro, M. (2018). Nested sequences of hippocampal assemblies during behavior support subsequent sleep replay. Science, 362(6415):675-679. Publisher: American Association for the Advancement of Science.

Drieu, C. and Zugaro, M. (2019). Hippocampal Sequences During Exploration: Mechanisms and Functions. Frontiers in Cellular Neuroscience, 13:232. 
Duncan, K., Ketz, N., Inati, S. J., and Davachi, L. (2012). Evidence for area CA1 as a match/mismatch detector: a high-resolution fMRI study of the human hippocampus. Hippocampus, 22(3):389-398. Publisher: Wiley Online Library.

Dunn, B., Mørreaunet, M., and Roudi, Y. (2015). Correlations and Functional Connections in a Population of Grid Cells. PLOS Computational Biology, 11(2):e1004052. Publisher: Public Library of Science.

Eichenbaum, H. (2017). On the Integration of Space, Time, and Memory. Neuron, 95(5):1007-1018.

Eliav, T., Maimon, S. R., Aljadeff, J., Tsodyks, M., Ginosar, G., Las, L., and Ulanovsky, N. (2021). Multiscale representation of very large environments in the hippocampus of flying bats. Science, 372(6545):eabg4020. Publisher: American Association for the Advancement of Science.

Feng, T., Silva, D., and Foster, D. J. (2015). Dissociation between the Experience-Dependent Development of Hippocampal Theta Sequences and Single-Trial Phase Precession. Journal of Neuroscience, 35(12):4890-4902. Publisher: Society for Neuroscience Section: Articles.

Fernández-Ruiz, A., Oliva, A., Nagy, G. A., Maurer, A. P., Berényi, A., and Buzsáki, G. (2017). Entorhinal-CA3 Dual-Input Control of Spike Timing in the Hippocampus by Theta-Gamma Coupling. Neuron, 93(5):12131226.e5.

Foster, D. J. and Wilson, M. A. (2006). Reverse replay of behavioural sequences in hippocampal place cells during the awake state. Nature, 440(7084):680-683. Bandiera_abtest: a Cg_type: Nature Research Journals Number: 7084 Primary_atype: Research Publisher: Nature Publishing Group.

Foster, D. J. and Wilson, M. A. (2007). Hippocampal theta sequences. Hippocampus, 17(11):1093-1099. _eprint: https://onlinelibrary.wiley.com/doi/pdf/10.1002/hipo.20345.

Gasparini, S., Migliore, M., and Magee, J. C. (2004). On the Initiation and Propagation of Dendritic Spikes in CA1 Pyramidal Neurons. Journal of Neuroscience, 24(49):11046-11056. Publisher: Society for Neuroscience Section: Cellular/Molecular.

Guardamagna, M., Eichler, R., Pedrosa, R., Aarts, A. A. A., Meyer, A. F., and Battaglia, F. P. (2021). The Hybrid Drive: a chronic implant device combining tetrode arrays with silicon probes for layer-resolved ensemble electrophysiology in freely moving mice. Technical report, bioRxiv. Company: Cold Spring Harbor Laboratory Distributor: Cold Spring Harbor Laboratory Label: Cold Spring Harbor Laboratory Section: New Results Type: article.

Gupta, A. S., van der Meer, M. A. A., Touretzky, D. S., and Redish, A. D. (2012). Segmentation of spatial experience by hippocampal theta sequences. Nature Neuroscience, 15(7):1032-1039. Bandiera_abtest: a Cg_type: Nature Research Journals Number: 7 Primary_atype: Research Publisher: Nature Publishing Group Subject_term: Hippocampus;Neural encoding;Spike-timing-dependent plasticity Subject_term_id: hippocampus;neural-encoding;spike-timing-dependent-plasticity. 
Harris, K. D., Henze, D. A., Csicsvari, J., Hirase, H., and Buzsáki, G. (2000). Accuracy of tetrode spike separation as determined by simultaneous intracellular and extracellular measurements. Journal of Neurophysiology, 84(1):401-414.

Harris, K. D., Henze, D. A., Hirase, H., Leinekugel, X., Dragoi, G., Czurkó, A., and Buzsáki, G. (2002). Spike train dynamics predicts theta-related phase precession in hippocampal pyramidal cells. Nature, 417(6890):738741.

Huxter, J., Burgess, N., and O'Keefe, J. (2003). Independent rate and temporal coding in hippocampal pyramidal cells. Nature, 425(6960):828-832. Bandiera_abtest: a Cg_type: Nature Research Journals Number: 6960 Primary_atype: Research Publisher: Nature Publishing Group.

Huxter, J. R., Senior, T. J., Allen, K., and Csicsvari, J. (2008). Theta phase-specific codes for two-dimensional position, trajectory and heading in the hippocampus. Nature Neuroscience, 11(5):587-594. Bandiera_abtest: a Cg_type: Nature Research Journals Number: 5 Primary_atype: Research Publisher: Nature Publishing Group.

Johnson, A. and Redish, A. D. (2007). Neural Ensembles in CA3 Transiently Encode Paths Forward of the Animal at a Decision Point. Journal of Neuroscience, 27(45):12176-12189. Publisher: Society for Neuroscience Section: Articles.

Kemere, C., Carr, M. F., Karlsson, M. P., and Frank, L. M. (2013). Rapid and Continuous Modulation of Hippocampal Network State during Exploration of New Places. PLOS ONE, 8(9):e73114. Publisher: Public Library of Science.

Kempter, R., Leibold, C., Buzsáki, G., Diba, K., and Schmidt, R. (2012). Quantifying circular-linear associations: Hippocampal phase precession. Journal of Neuroscience Methods, 207(1):113-124.

Klausberger, T. and Somogyi, P. (2008). Neuronal Diversity and Temporal Dynamics: The Unity of Hippocampal Circuit Operations. Science, 321(5885):53-57. Publisher: American Association for the Advancement of Science.

Lasztóczi, B. and Klausberger, T. (2014). Layer-Specific GABAergic Control of Distinct Gamma Oscillations in the CA1 Hippocampus. Neuron, 81(5):1126-1139.

Lasztóczi, B. and Klausberger, T. (2016). Hippocampal Place Cells Couple to Three Different Gamma Oscillations during Place Field Traversal. Neuron, 91(1):34-40.

Lee, A. K. and Wilson, M. A. (2002). Memory of sequential experience in the hippocampus during slow wave sleep. Neuron, 36(6):1183-1194.

Leão, R. N., Mikulovic, S., Leão, K. E., Munguba, H., Gezelius, H., Enjin, A., Patra, K., Eriksson, A., Loew, L. M., Tort, A. B., and Kullander, K. (2012). OLM interneurons differentially modulate CA3 and entorhinal inputs to hippocampal CA1 neurons. Nature neuroscience, 15(11):1524-1530. 
Lisman, J. and Redish, A. (2009). Prediction, sequences and the hippocampus. Philosophical Transactions of the Royal Society B: Biological Sciences, 364(1521):1193-1201. Publisher: Royal Society.

Markram, H., Lubke, J., Frotscher, M., and Sakmann, B. (1997). Regulation of synaptic efficacy by coincidence of postsynaptic APs and EPSPs. Science, 275(5297):213-5.

McClelland, J. L., McNaughton, B. L., and O'Reilly, R. C. (1995). Why there are complementary learning systems in the hippocampus and neocortex: insights from the successes and failures of connectionist models of learning and memory. Psychological Review, 102(3):419-57.

McNaughton, B. L., Battaglia, F. P., Jensen, O., Moser, E. I., and Moser, M.-B. (2006). Path integration and the neural basis of the 'cognitive map'. Nature Reviews Neuroscience, 7(8):663-678. Bandiera_abtest: a Cg_type: Nature Research Journals Number: 8 Primary_atype: Reviews Publisher: Nature Publishing Group.

Mehta, M. R., Lee, A. K., and Wilson, M. A. (2002). Role of experience and oscillations in transforming a rate code into a temporal code. Nature, 417(6890):741-746.

Mehta, M. R., Quirk, M. C., and Wilson, M. A. (2000). Experience-Dependent Asymmetric Shape of Hippocampal Receptive Fields. Neuron, 25(3):707-715.

Middleton, S. J. and McHugh, T. J. (2016). Silencing CA3 disrupts temporal coding in the CA1 ensemble. Nature Neuroscience, 19(7):945-951. Bandiera_abtest: a Cg_type: Nature Research Journals Number: 7 Primary_atype: Research Publisher: Nature Publishing Group Subject_term: Hippocampus;Neural circuits;Neuronal physiology Subject_term_id: hippocampus;neural-circuit;neuronal-physiology.

Milstein, A. D., Li, Y., Bittner, K. C., Grienberger, C., Soltesz, I., Magee, J. C., and Romani, S. (2021). Bidirectional synaptic plasticity rapidly modifies hippocampal representations. Technical report, bioRxiv. Company: Cold Spring Harbor Laboratory Distributor: Cold Spring Harbor Laboratory Label: Cold Spring Harbor Laboratory Section: New Results Type: article.

Mizuseki, K., Diba, K., Pastalkova, E., and Buzsáki, G. (2011). Hippocampal CA1 pyramidal cells form functionally distinct sublayers. Nature Neuroscience, 14(9):1174-1181. Bandiera_abtest: a Cg_type: Nature Research Journals Number: 9 Primary_atype: Research Publisher: Nature Publishing Group Subject_term: Cellular neuroscience;Neuronal physiology;Sleep Subject_term_id: cellular-neuroscience;neuronal-physiology;sleep.

Mizuseki, K., Sirota, A., Pastalkova, E., and Buzsáki, G. (2009). Theta Oscillations Provide Temporal Windows for Local Circuit Computation in the Entorhinal-Hippocampal Loop. Neuron, 64(2):267-280.

Montgomery, S. M., Betancur, M. I., and Buzsáki, G. (2009). Behavior-Dependent Coordination of Multiple Theta Dipoles in the Hippocampus. Journal of Neuroscience, 29(5):1381-1394. Publisher: Society for Neuroscience Section: Articles. 
Moser, E. I., Kropff, E., and Moser, M.-B. (2008). Place Cells, Grid Cells, and the Brain's Spatial Representation System. Annual Review of Neuroscience, 31(1):69-89._ _eprint: https://doi.org/10.1146/annurev.neuro.31.061307.090723.

Navas-Olive, A., Valero, M., Jurado-Parras, T., Salas-Quiroga, A. d., Averkin, R. G., Gambino, G., Cid, E., and Prida, L. M. d. l. (2020). Multimodal determinants of phase-locked dynamics across deep-superficial hippocampal sublayers during theta oscillations. Nature Communications.

Nicholson, C. and Freeman, J. A. (1975). Theory of current source-density analysis and determination of conductivity tensor for anuran cerebellum. Journal of Neurophysiology, 38(2):356-368.

O'Keefe, J. and Recce, M. L. (1993). Phase relationship between hippocampal place units and the EEG theta rhythm. Hippocampus, 3(3):317-330._eprint: https://onlinelibrary.wiley.com/doi/pdf/10.1002/hipo.450030307.

Pettersen, K. H., Devor, A., Ulbert, I., Dale, A. M., and Einevoll, G. T. (2006). Current-source density estimation based on inversion of electrostatic forward solution: Effects of finite extent of neuronal activity and conductivity discontinuities. Journal of Neuroscience Methods, 154(1):116-133.

Pfeiffer, B. E. and Foster, D. J. (2013). Hippocampal place-cell sequences depict future paths to remembered goals. Nature, 497(7447):74-79. Bandiera_abtest: a Cg_type: Nature Research Journals Number: 7447 Primary_atype: Research Publisher: Nature Publishing Group Subject_term: Decision;Hippocampus;Learning and memory;Neurophysiology Subject_term_id: decision;hippocampus;learning-and-memory;neurophysiology.

Pfeiffer, B. E. and Foster, D. J. (2015). Autoassociative dynamics in the generation of sequences of hippocampal place cells. Science, 349(6244):180-183. Publisher: American Association for the Advancement of Science.

Quiroga, R. Q. and Panzeri, S. (2013). Principles of Neural Coding. CRC Press. Google-Books-ID: MkTNBQAAQBAJ.

Reifenstein, E. T., Bin Khalid, I., and Kempter, R. (2021). Synaptic learning rules for sequence learning. eLife, 10:e67171. Publisher: eLife Sciences Publications, Ltd.

Remondes, M. and Schuman, E. M. (2002). Direct cortical input modulates plasticity and spiking in CA1 pyramidal neurons. Nature, 416(6882):736-740. Bandiera_abtest: a Cg_type: Nature Research Journals Number: 6882 Primary_atype: Research Publisher: Nature Publishing Group.

Renoult, L., Irish, M., Moscovitch, M., and Rugg, M. D. (2019). From Knowing to Remembering: The Semantic-Episodic Distinction. Trends in Cognitive Sciences, 23(12):1041-1057.

Schlesiger, M. I., Cannova, C. C., Boublil, B. L., Hales, J. B., Mankin, E. A., Brandon, M. P., Leutgeb, J. K., Leibold, C., and Leutgeb, S. (2015). The medial entorhinal cortex is necessary for temporal organization of hippocampal neuronal activity. Nature Neuroscience, 18(8):1123-1132. Bandiera_abtest: a Cg_type: Nature 
Research Journals Number: 8 Primary_atype: Research Publisher: Nature Publishing Group Subject_term: Neural circuits Subject_term_id: neural-circuit.

Schmidt, R., Diba, K., Leibold, C., Schmitz, D., Buzsáki, G., and Kempter, R. (2009). Single-Trial Phase Precession in the Hippocampus. Journal of Neuroscience, 29(42):13232-13241. Publisher: Society for Neuroscience Section: Articles.

Schomburg, E. W., Fernández-Ruiz, A., Mizuseki, K., Berényi, A., Anastassiou, C. A., Koch, C., and Buzsáki, G. (2014). Theta Phase Segregation of Input-Specific Gamma Patterns in Entorhinal-Hippocampal Networks. Neuron, 84(2):470-485. Publisher: Elsevier.

Sharif, F., Tayebi, B., Buzsáki, G., Royer, S., and Fernandez-Ruiz, A. (2021). Subcircuits of Deep and Superficial CA1 Place Cells Support Efficient Spatial Coding across Heterogeneous Environments. Neuron, 109(2):363376.e6.

Shepherd, G. M., editor (2004). The Synaptic Organization of the Brain. Oxford University Press, New York, 5 edition.

Siegle, J. H., López, A. C., Patel, Y. A., Abramov, K., Ohayon, S., and Voigts, J. (2017). Open Ephys: an open-source, plugin-based platform for multichannel electrophysiology. Journal of Neural Engineering, 14(4):045003. Publisher: IOP Publishing.

Skaggs, W. E. and McNaughton, B. L. (1996). Replay of Neuronal Firing Sequences in Rat Hippocampus During Sleep Following Spatial Experience. Science, 271(5257):1870-1873. Publisher: American Association for the Advancement of Science.

Skaggs, W. E., McNaughton, B. L., Wilson, M. A., and Barnes, C. A. (1996). Theta phase precession in hippocampal neuronal populations and the compression of temporal sequences. Hippocampus, 6(2):149-172. _ _ eprint: https://onlinelibrary.wiley.com/doi/pdf/10.1002/\%28SICI\%2910981063\%281996\%296\%3A2\%3C149\%3A\%3AAID-HIPO6\%3E3.0.CO\%3B2-K.

Squire, L. R. and Knowlton, B. J. (1995). Memory, hippocampus, and brain systems. In The cognitive neurosciences, pages 825-837. The MIT Press, Cambridge, MA, US.

Takahashi, M., Nishida, H., David Redish, A., and Lauwereyns, J. (2014). Theta phase shift in spike timing and modulation of gamma oscillation: a dynamic code for spatial alternation during fixation in rat hippocampal area CA1. Journal of Neurophysiology, 111(8):1601-1614. Publisher: American Physiological Society.

Teyler, T. J. and DiScenna, P. (1986). The hippocampal memory indexing theory. Behav Neurosci, 100(2):14754.

Treves, A. and Rolls, E. T. (1994). Computational analysis of the role of the hippocampus in memory. Hippocampus, 4(3):374-391. _ eprint: https://onlinelibrary.wiley.com/doi/pdf/10.1002/hipo.450040319. 
Tsodyks, M. V., Skaggs, W. E., Sejnowski, T. J., and McNaughton, B. L. (1996). Population dynamics and theta rhythm phase precession of hippocampal place cell firing: A spiking neuron model. Hippocampus, 6(3):271-280. _ eprint: https://onlinelibrary.wiley.com/doi/pdf/10.1002/\%28SICI\%2910981063\%281996\%296\%3A3\%3C271\%3A\%3AAID-HIPO5\%3E3.0.CO\%3B2-Q.

Tukker, J. J., Lasztóczi, B., Katona, L., Roberts, J. D. B., Pissadaki, E. K., Dalezios, Y., Márton, L., Zhang, L., Klausberger, T., and Somogyi, P. (2013). Distinct Dendritic Arborization and In Vivo Firing Patterns of Parvalbumin-Expressing Basket Cells in the Hippocampal Area CA3. Journal of Neuroscience, 33(16):68096825. Publisher: Society for Neuroscience Section: Articles.

Valero, M., Cid, E., Averkin, R. G., Aguilar, J., Sanchez-Aguilera, A., Viney, T. J., Gomez-Dominguez, D., Bellistri, E., and de la Prida, L. M. (2015). Determinants of different deep and superficial CA1 pyramidal cell dynamics during sharp-wave ripples. Nature Neuroscience, 18(9):1281-1290. Bandiera_abtest: a Cg_type: Nature Research Journals Number: 9 Primary_atype: Research Publisher: Nature Publishing Group Subject_term: Inhibition-excitation balance;Neural circuits Subject_term_id: inhibition-excitationbalance;neural-circuit.

Valero, M. and de la Prida, L. M. (2018). The hippocampus in depth: a sublayer-specific perspective of entorhinal-hippocampal function. Current Opinion in Neurobiology, 52:107-114.

Vinogradova, O. S. (2001). Hippocampus as comparator: role of the two input and two output systems of the hippocampus in selection and registration of information. Hippocampus, 11(5):578-598. Publisher: Wiley Online Library.

Wang, M., Foster, D. J., and Pfeiffer, B. E. (2020). Alternating sequences of future and past behavior encoded within hippocampal theta oscillations. Science, 370(6513):247-250. Publisher: American Association for the Advancement of Science.

Wikenheiser, A. M. and Redish, A. D. (2015). Hippocampal theta sequences reflect current goals. Nature Neuroscience, 18(2):289-294. Bandiera_abtest: a Cg_type: Nature Research Journals Number: 2 Primary_atype: Research Publisher: Nature Publishing Group Subject_term: Decision;Hippocampus;Navigation Subject_term_id: decision;hippocampus;navigation.

Witter, M. P. and Moser, E. I. (2006). Spatial representation and the architecture of the entorhinal cortex. Trends in Neurosciences, 29(12):671-678.

Yamaguchi, Y., Aota, Y., McNaughton, B. L., and Lipa, P. (2002). Bimodality of theta phase precession in hippocampal place cells in freely running rats. J Neurophysiol, 87(6):2629-42.

Zhang, K., Ginzburg, I., McNaughton, B. L., and Sejnowski, T. J. (1998). Interpreting Neuronal Population Activity by Reconstruction: Unified Framework With Application to Hippocampal Place Cells. Journal of Neurophysiology, 79(2):1017-1044. Publisher: American Physiological Society. 
bioRxiv preprint doi: https://doi.org/10.1101/2021.12.22.473863; this version posted December 23, 2021. The copyright holder for this preprint (which was not certified by peer review) is the author/funder, who has granted bioRxiv a license to display the preprint in perpetuity. It is made available under aCC-BY-NC-ND 4.0 International license.

Ólafsdóttir, H. F., Bush, D., and Barry, C. (2018). The Role of Hippocampal Replay in Memory and Planning. Current Biology, 28(1):R37-R50. 
bioRxiv preprint doi: https://doi.org/10.1101/2021.12.22 473863; this version posted December 23, 2021. The copyright holder for this

preprint (which was not certified by peer review) is the author/funder, who has granted bioRxiv a license to display the preprint in perpetuity. It is made available under aCC-BY-NC-ND 4.0 International license.

\section{Supplementary Figures}

A

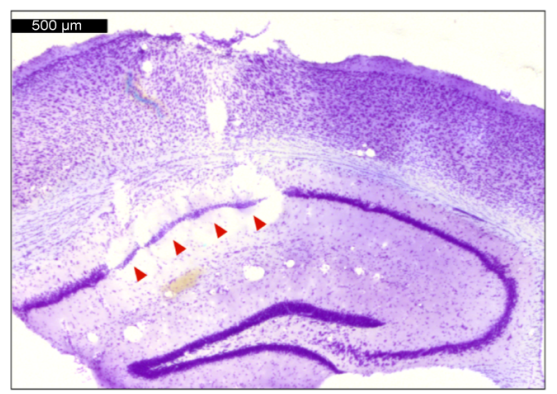

$\mathrm{D}$

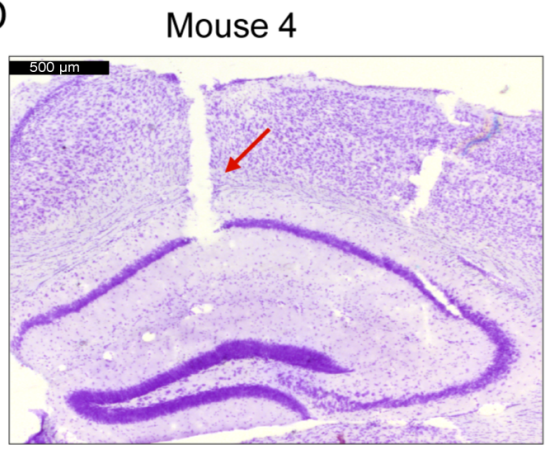

B

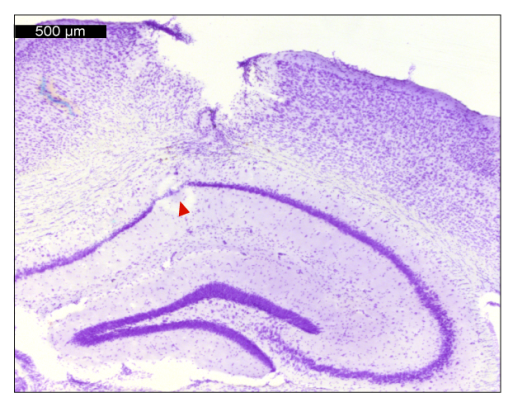

E

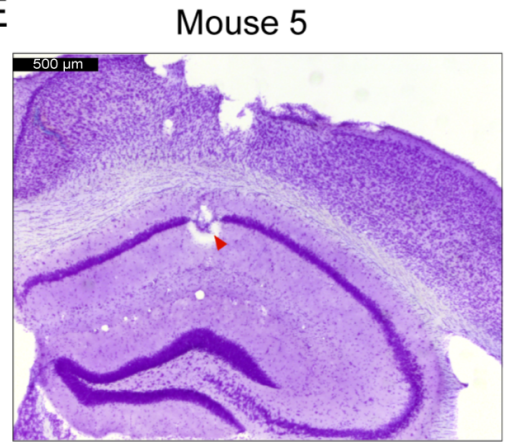

C

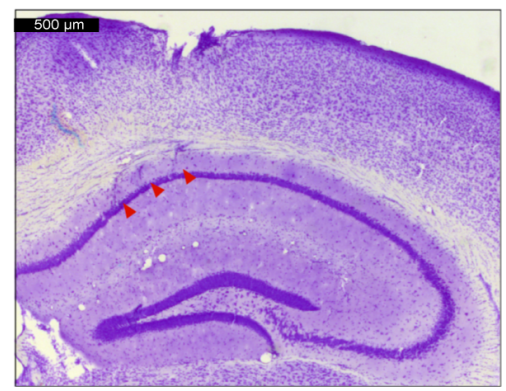

$\mathrm{F}$

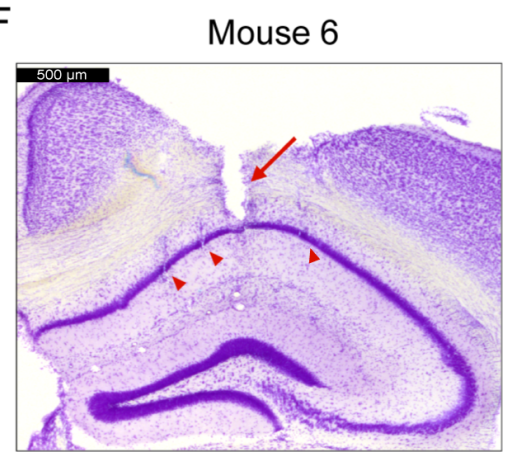

Figure S1: Histological verification of tetrodes and silicon probe position. Representative Nissl-stained coronal section showing example recording locations from each animal. Red triangles indicate the estimated location of tetrode tips (CA1 pyramidal layer), while red arrows indicate the trace of the silicon probe shaft. Mouse 1, 2 and 5 received electrolytic lesions at the end of the experiment. Mouse 3, 4 and 6 never electrolytic lesions, to better visualize the silicon probe track. Scale bar denote $0.5 \mathrm{~mm}$. 


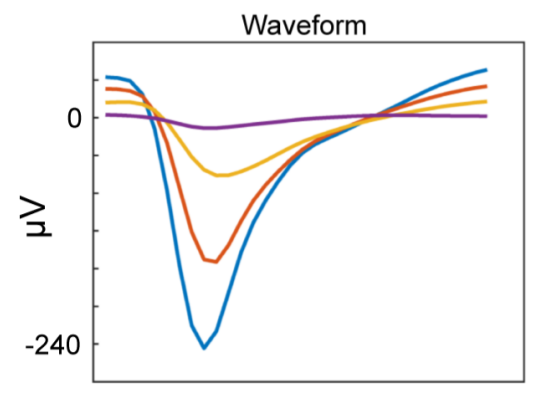

Mouse 1, S1 Cell 41 (Phase precessing)
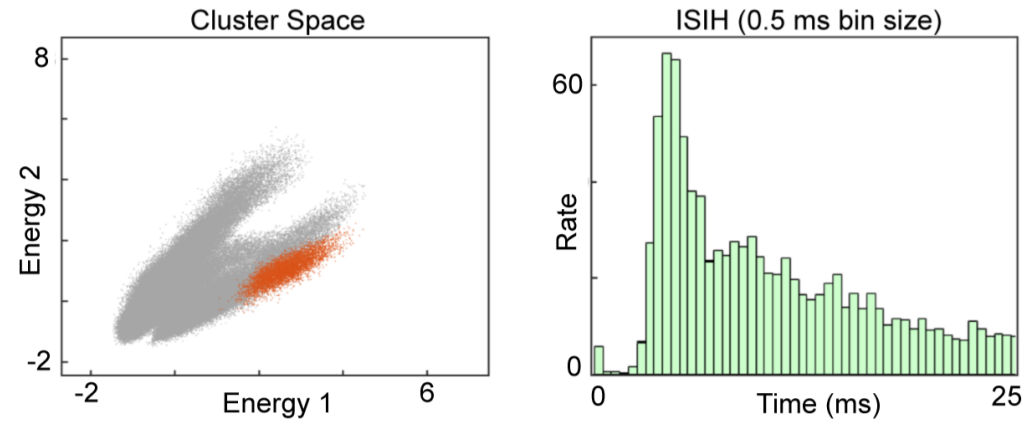

\section{Mouse 1, S1 Cell 68 (Phase locking)}
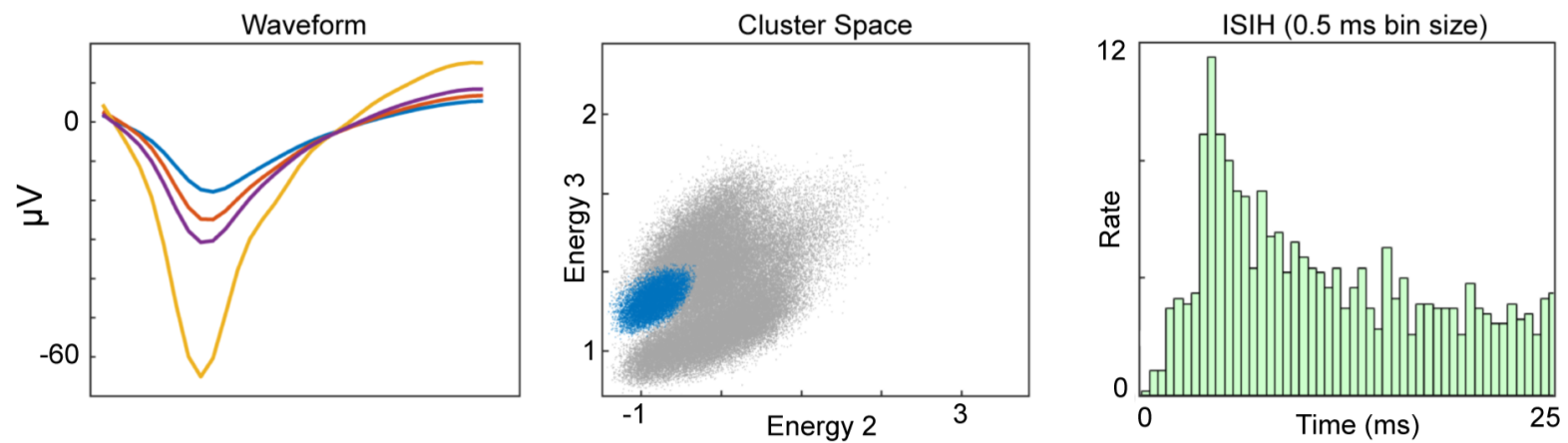

Mouse 6, S1 Cell 28 (Phase precessing)
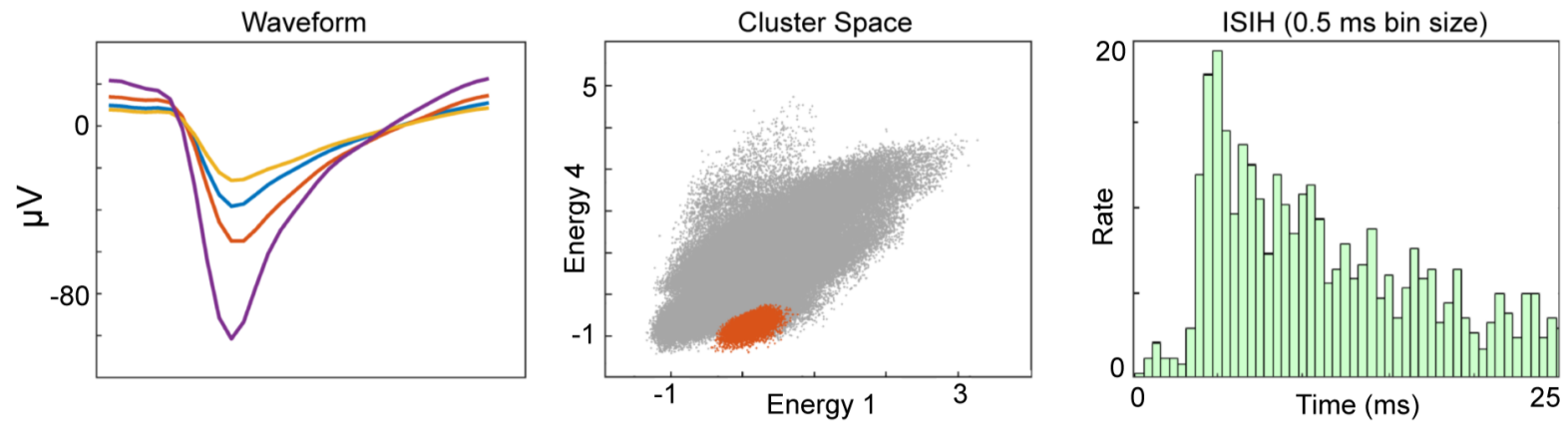

Mouse 6, S1 Cell 103 (Phase locking)
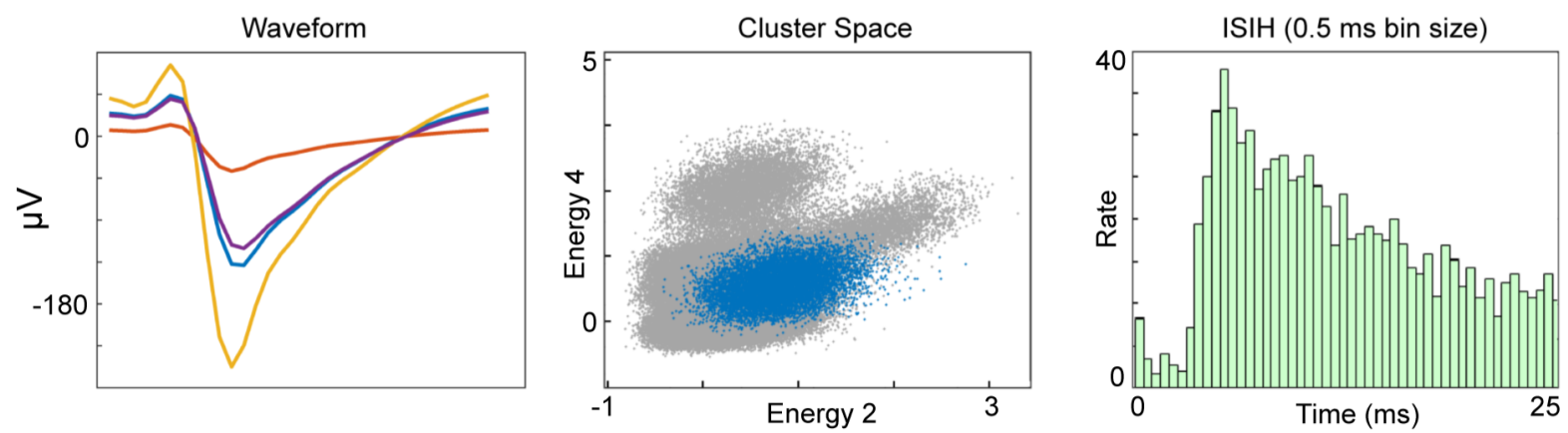

Figure S2: Cluster information of individual single unit examples (Figure 2 $\mathrm{C}$ ). Information for the individual units reported in Figure 2C. Left panel, mean waveform across the 4 channels of the tetrode. Center panel, 2D cluster projection of the recorded spikes. The cell's cluster is highlighted by blue or orange color. Right panel, interspike-interval histogram (0.5 ms time bins). 
bioRxiv preprint doi: https://doi.org/10.1101/2021.12.22 473863; this version posted December 23, 2021. The copyright holder for this preprint (which was not certified by peer review) is the author/funder, who has granted bioRxiv a license to display the preprint in perpetuity. It is made available under aCC-BY-NC-ND 4.0 International license.

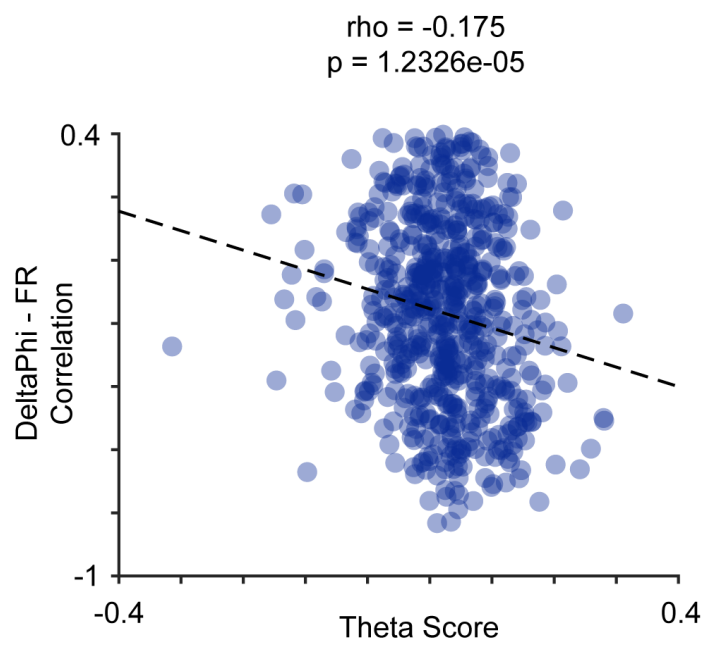

Figure S3: Relationship of Theta Score and DeltaPhi-Firing Rate correlation Correlation between the Theta Score and the DeltaPhi - Firing Rate (FR) correlation for field one of each cell ( $\mathrm{p}<0.01$; Spearman's Rho $=-0.175)$.
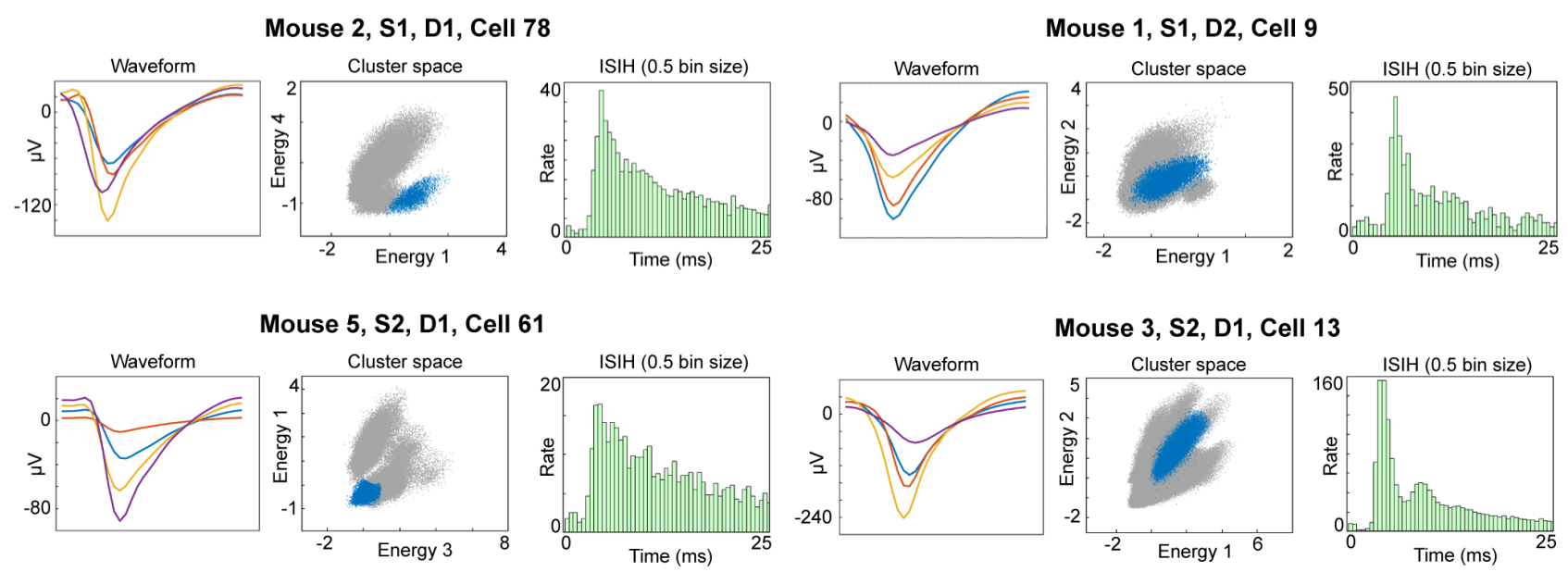

Mouse 3, S2, D1, Cell 13
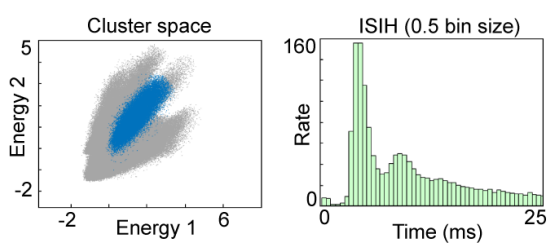

Mouse 1, S2, Cell 20
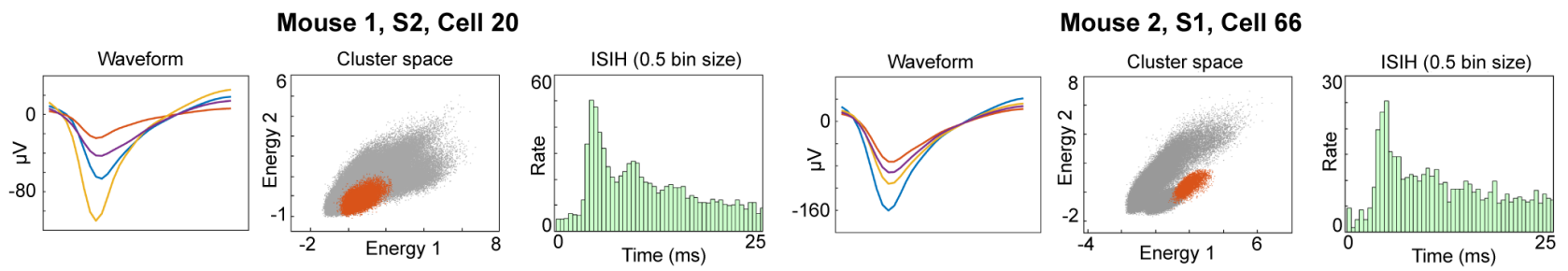

Figure S4: Cluster information of individual single unit examples (Figure 3C,F). Information for the individual units reported in Figure $3 \mathrm{C}, \mathrm{F}$. Left panel, mean waveform across the 4 channels of the tetrode. Center panel, 2D cluster projection of the recorded spikes. The cell's cluster is highlighted by blue or orange color. Right panel, interspike-interval histogram (0.5 ms time bins). 
bioRxiv preprint doi: https://doi.org/10.1101/2021.12.22.473863; this version posted December 23, 2021. The copyright holder for this preprint (which was not certified by peer review) is the author/funder, who has granted bioRxiv a license to display the preprint in perpetuity. It is made available under aCC-BY-NC-ND 4.0 International license.

\section{A}
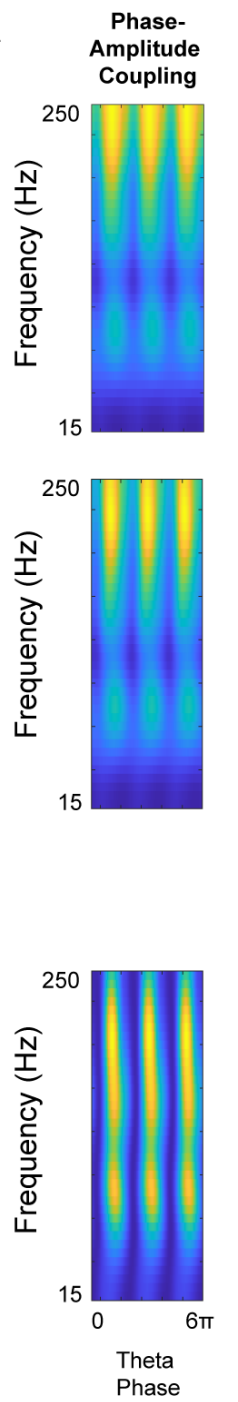
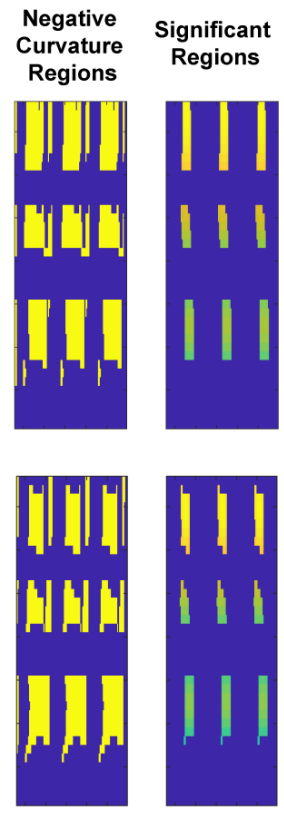

$\bullet \bullet \bullet$
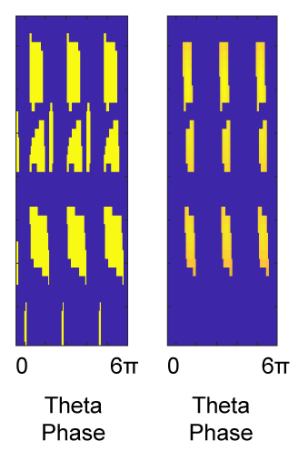
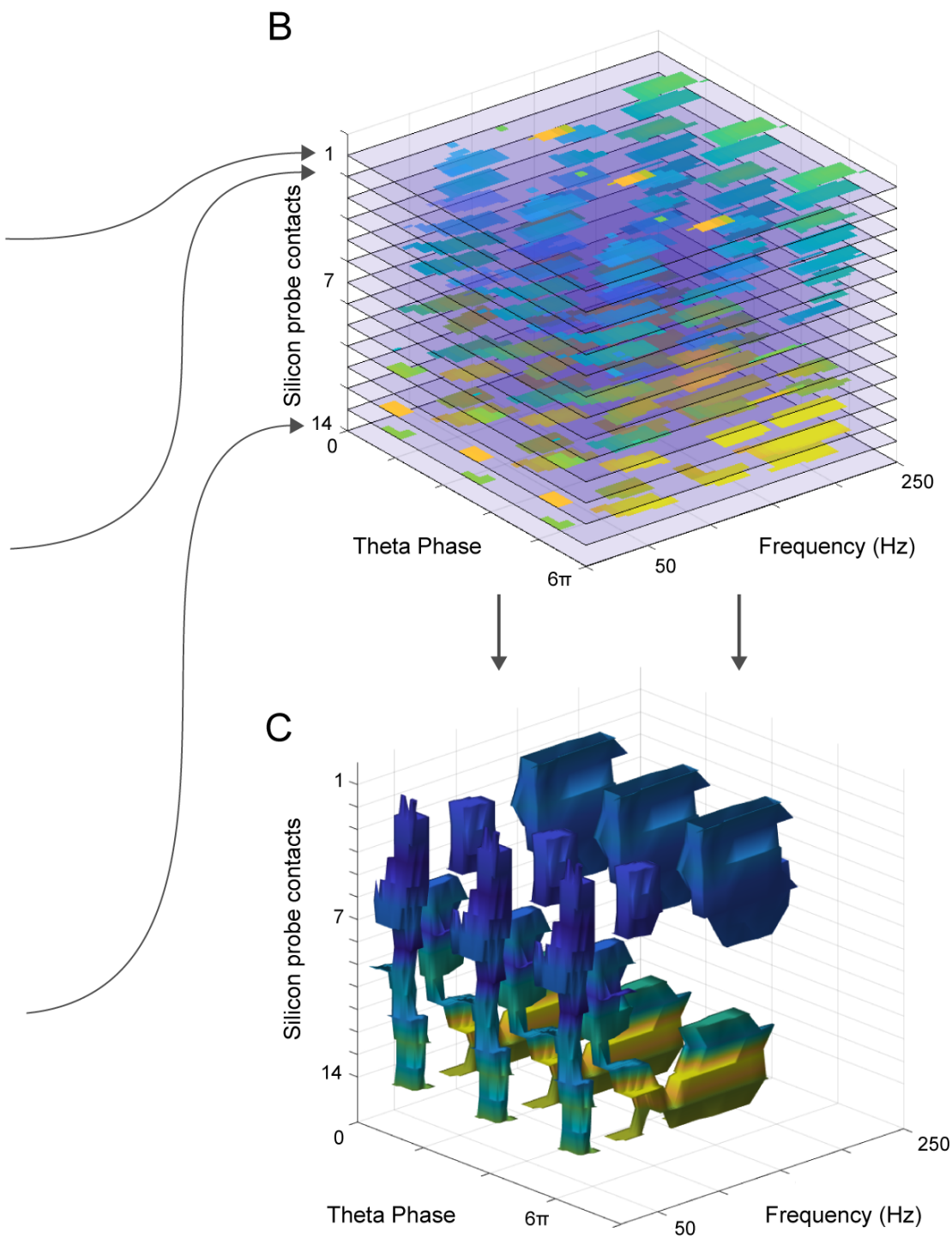

Figure S5: Construction of a 3D phase-amplitude-depth structure to visualize oscillatory interactions at different frequencies. (A) Left panel, phase-amplitude plot between the theta oscillation (6-10 $\mathrm{Hz})$ and the power of oscillations at higher frequencies (15-250 Hz). Center panel, negative curvature regions found applying a discrete laplace operator to the frequency-phase matrix obtained in left panel. Right panel, matrix with further selected bins that showed a coupling strength above 1STD of the mean of the coupling of the respective frequency. Each analysis was repeated for each CSD-derived signal from 14 contacts of the silicon probe (16 minus the 2 extremes). (B) Stacked matrices with bins that showed a coupling strength above 1STD of the mean of the coupling of the respective frequency (same as in right panel (A)). (C) 3D Basins structure obtained concatenating the significant coupling values in Panel (B) at different depths. 
bioRxiv preprint doi: https://doi.org/10.1101/2021.12.22.473863; this version posted December 23, 2021. The copyright holder for this preprint (which was not certified by peer review) is the author/funder, who has granted bioRxiv a license to display the preprint in perpetuity. It is made available under aCC-BY-NC-ND 4.0 International license.
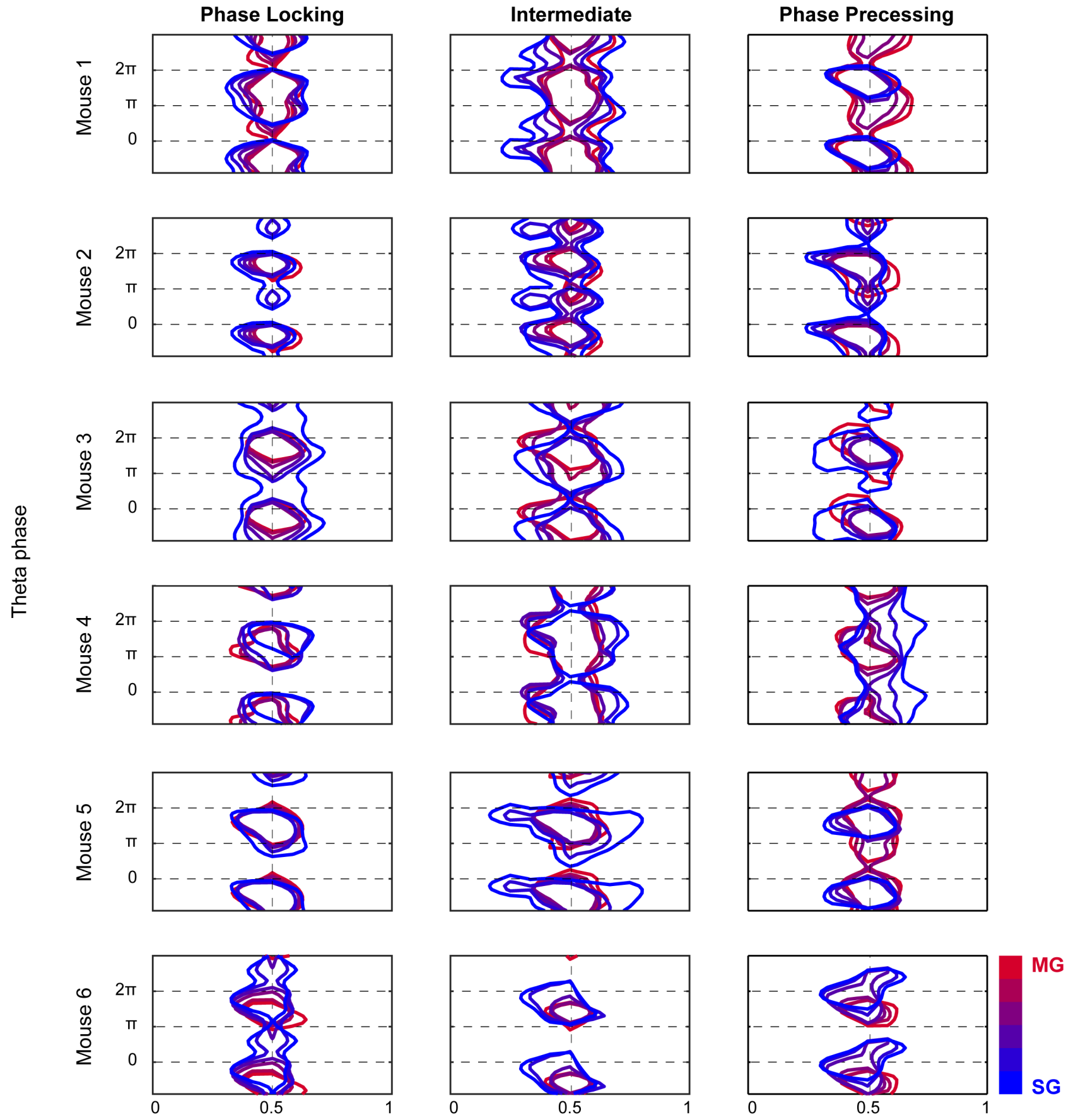

In-field position

Figure S6: Spike density contour plot for each animal. Contour plot of the GLM-derived spike density plot (same as in 5B) for each animal, as a function of the slow-medium gamma coefficient. 
bioRxiv preprint doi: https://doi.org/10.1101/2021.12.22.473863; this version posted December 23, 2021. The copyright holder for this preprint (which was not certified by peer review) is the author/funder, who has granted bioRxiv a license to display the preprint in perpetuity. It is made available under aCC-BY-NC-ND 4.0 International license.
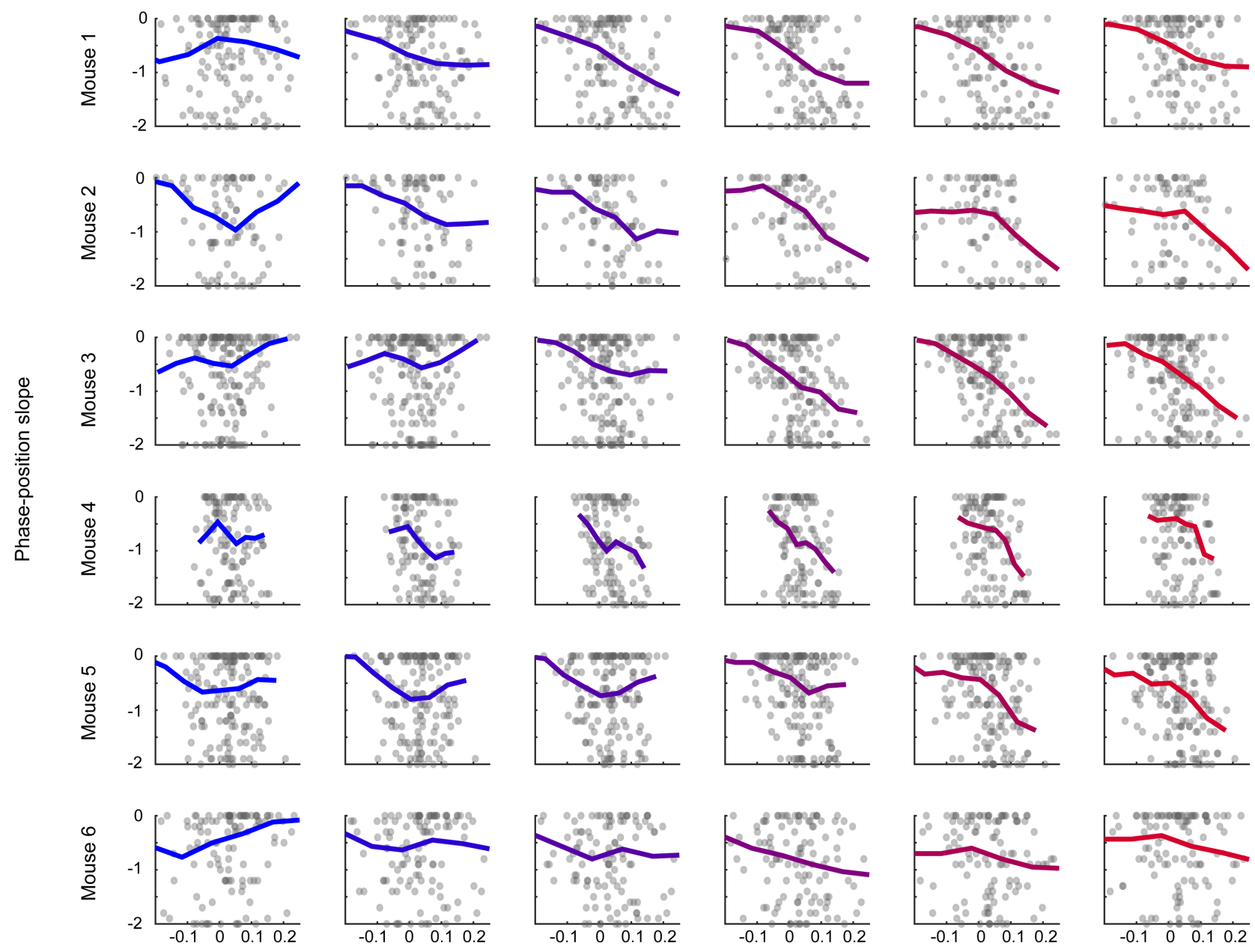

Theta score

slow gamma

medium gamma

Figure S7: Single cell regression plots for each animal. Phase-position slope values plotted against the Theta Score values for each individual cell, as a function of slow-medium gamma balance, for each animal. 
bioRxiv preprint doi: https://doi.org/10.1101/2021.12.22.473863; this version posted December 23, 2021. The copyright holder for this preprint (which was not certified by peer review) is the author/funder, who has granted bioRxiv a license to display the preprint in perpetuity. It is made available under aCC-BY-NC-ND 4.0 International license.

A

Velocity

intervals $(\mathrm{cm} / \mathrm{s})$

$[0.5-9]$
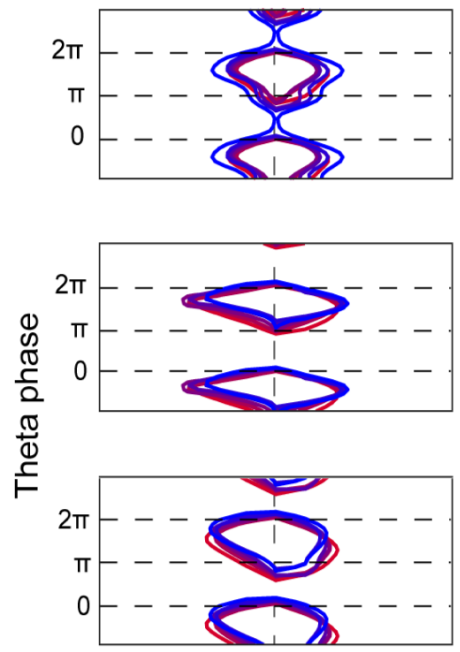

[26 - 35]

\section{Phase Locking}

$[9-17]$

[17 - 26]

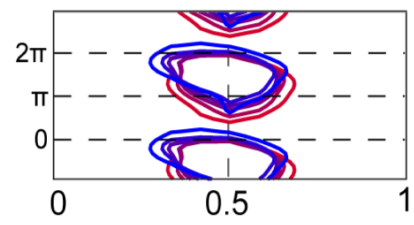

Intermediate
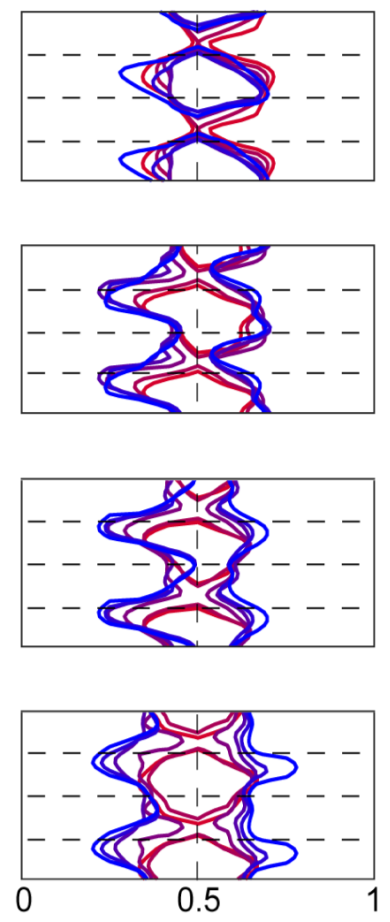

In-field position

\section{Phase Precessing}
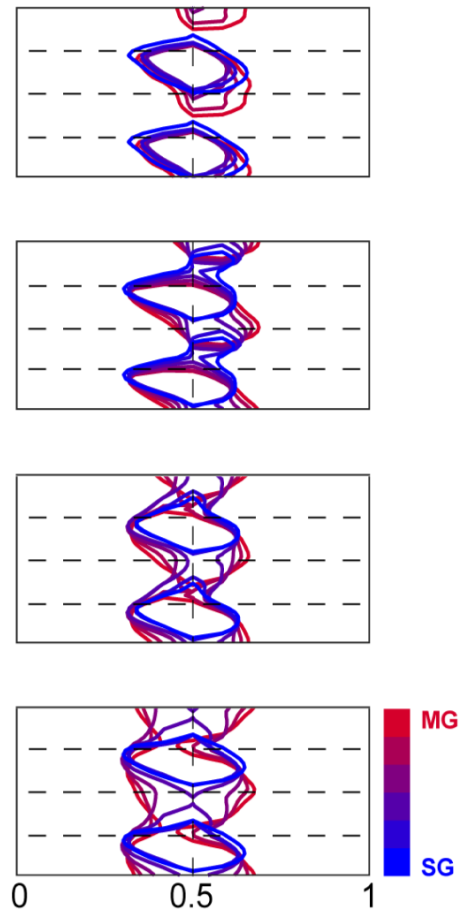

Figure S8: Spike density contour plot using velocity as an additional factor in the GLM. Contour plot of the GLM-derived spike density plot as a function of velocity. 
bioRxiv preprint doi: https://doi.org/10.1101/2021.12.22.473863; this version posted December 23, 2021. The copyright holder for this preprint (which was not certified by peer review) is the author/funder, who has granted bioRxiv a license to display the preprint in perpetuity. It is made available under aCC-BY-NC-ND 4.0 International license.

A

Preferred phase probability (REM)

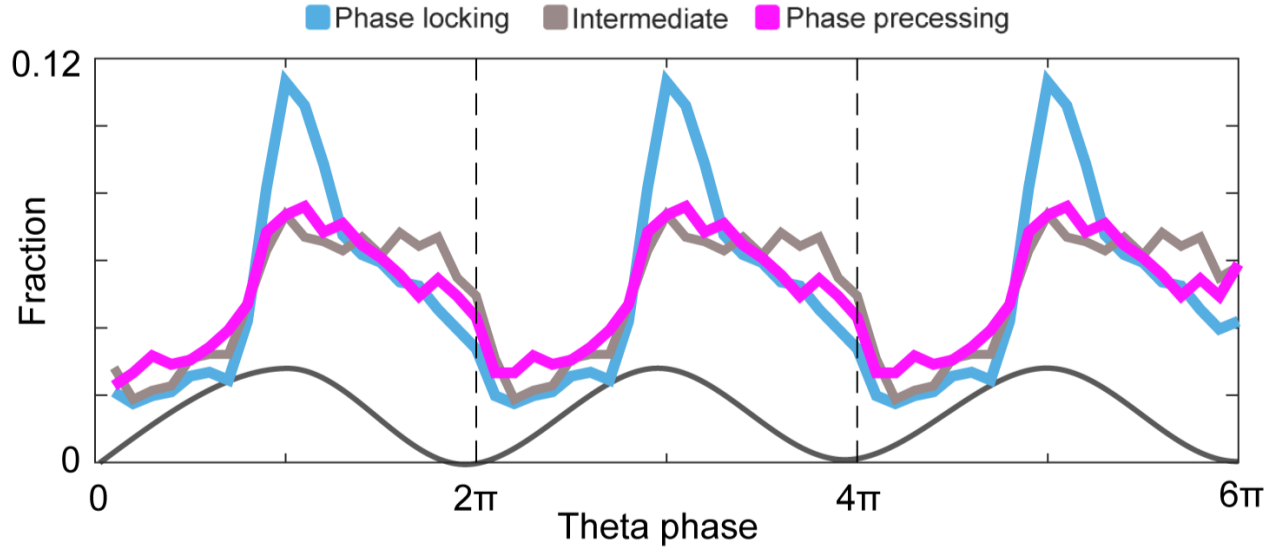

B

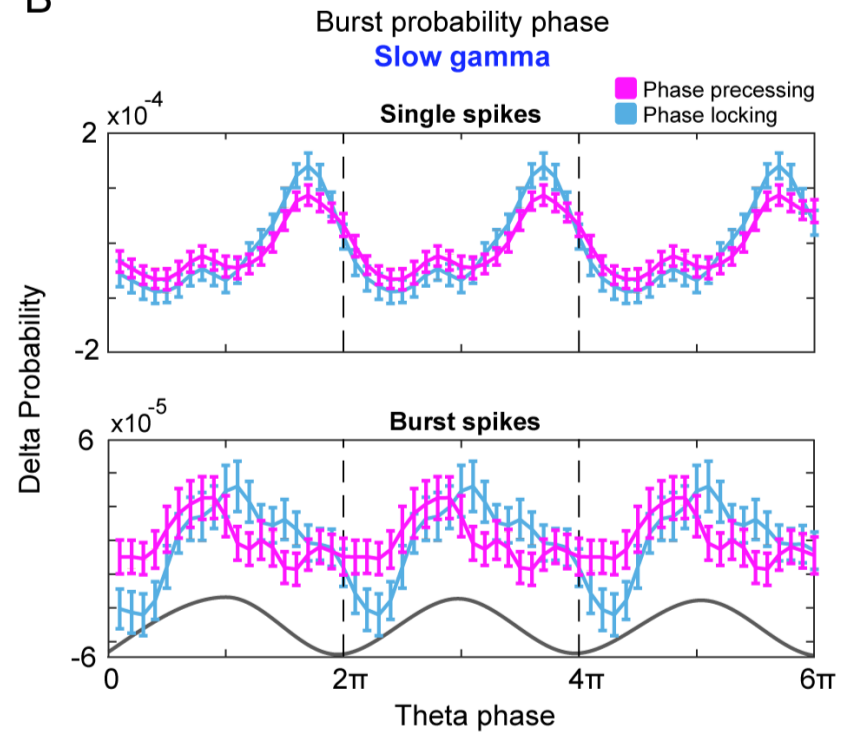

C

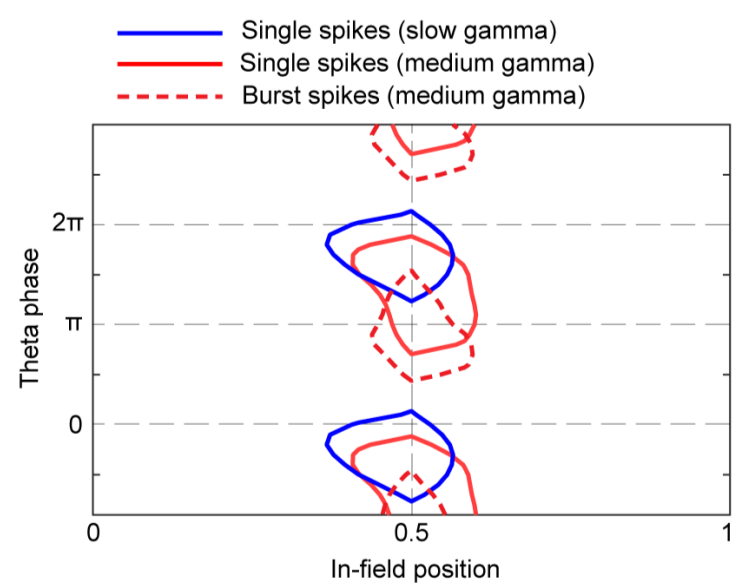

Figure S9: Supplementary information to Figure 5. (A) Preferred firing phase preferences for phase precessing, intermediate and phase locking cells during REM sleep. (B) Phase distributions of single spikes and burst spikes during slow gamma dominated periods, for phase locking (light blue) and phase precessing (violet) cells. (C) Contour of regions with highest density of spikes for single spikes during slow gamma (solid blue line), for single spikes during medium gamma (solid red line) and for burst spikes during medium gamma (dashed red line). 
A

Phase locking

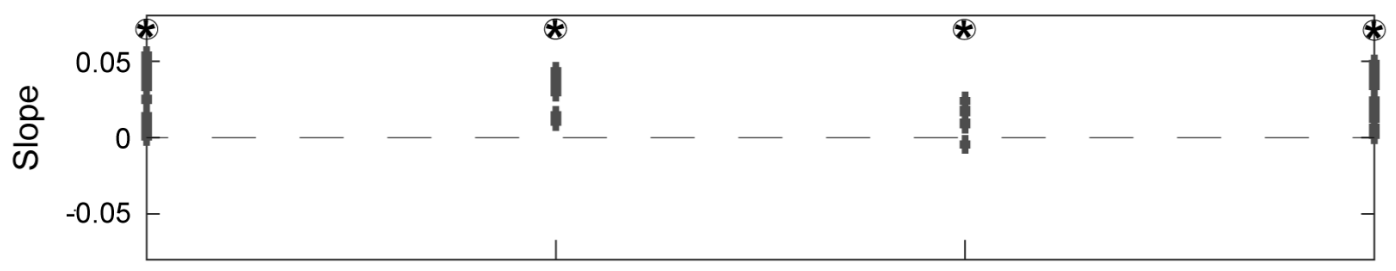

Phase precessing

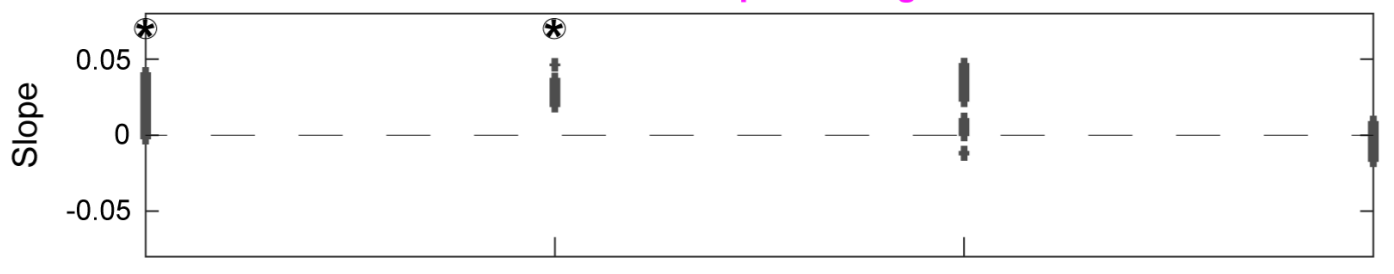

slow gamma

medium gamma

B

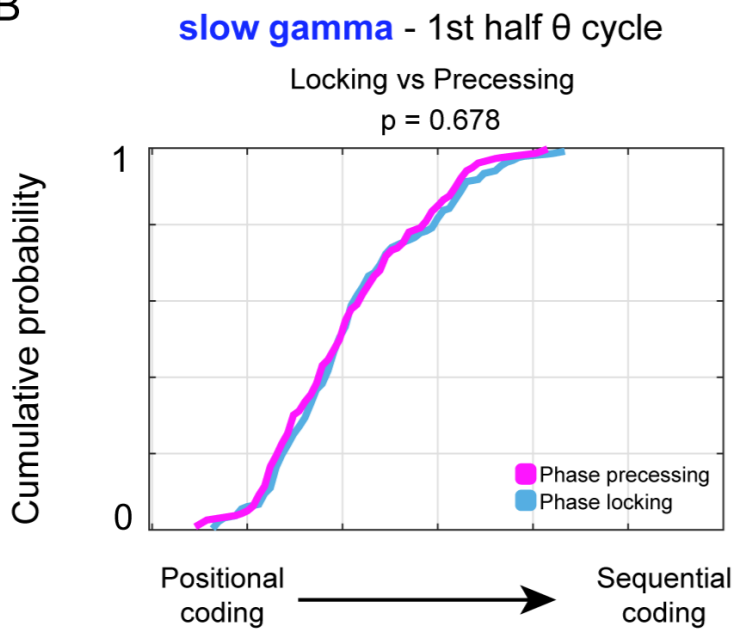

slow gamma - 2 nd half $\theta$ cycle Locking vs Precessing $p=0.103$

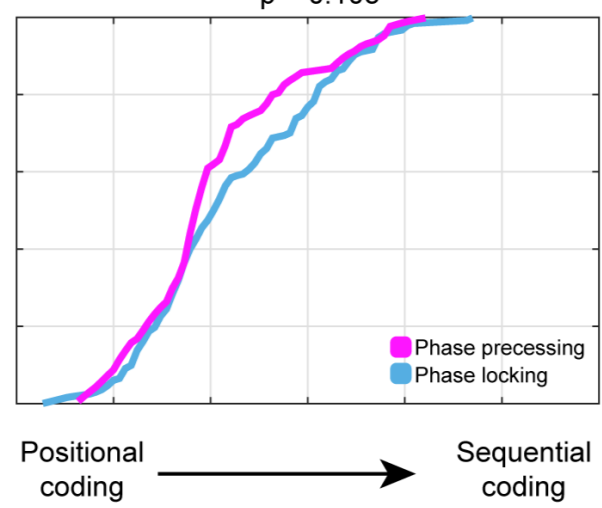

Figure S10: Supplementary information to Figure 6. (A) Place field distance vs phase distance regression slope for different gamma coefficients and populations, for each animal. Significant point are highlighted with an asterisk ( $\mathrm{p}<0.05$; t-test). (B) Both in the first and second half of slow gamma dominated theta cycles, phase precessing and phase locking encode a similar amount of sequential information ( $p>0.05$; Two-sample Kolmogorov-Smirnov test). 
A

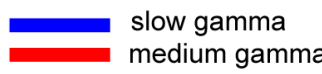

Phase Locking

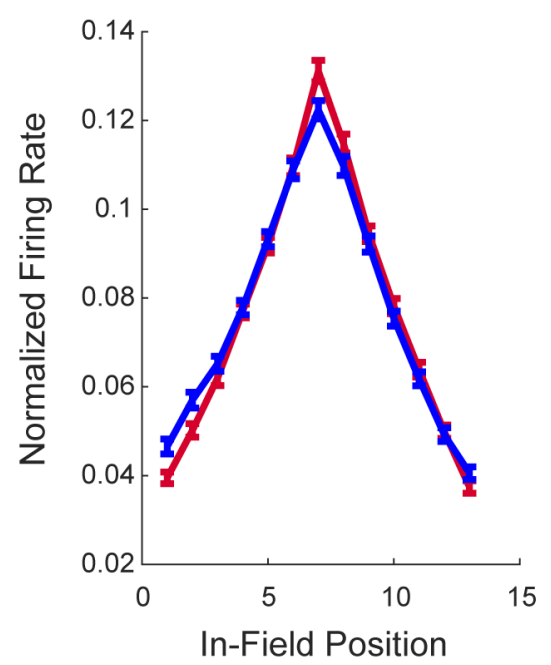

B

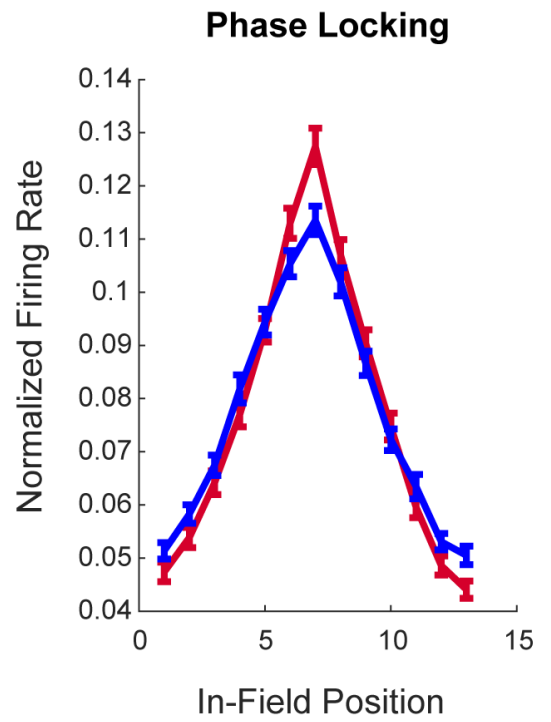

\section{Single Spikes}

Intermediate

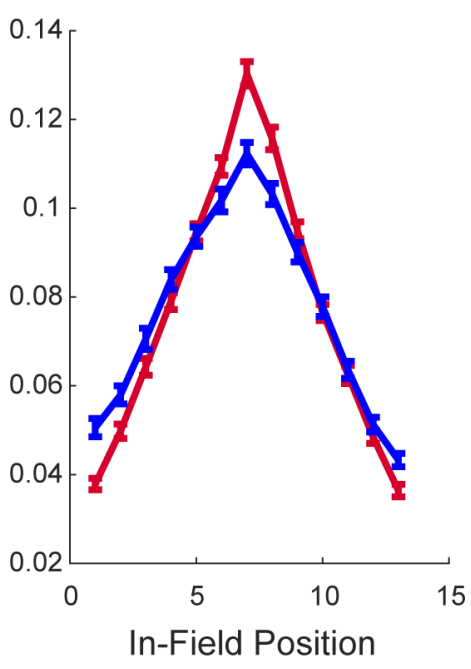

Burst Spikes

Intermediate

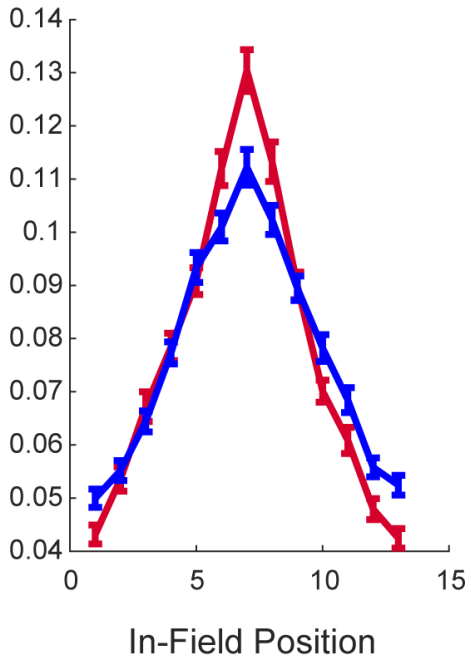

Phase Precessing

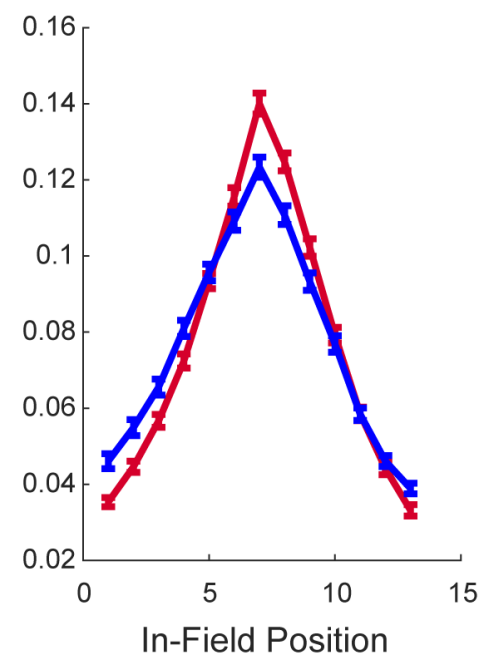

Phase Precessing

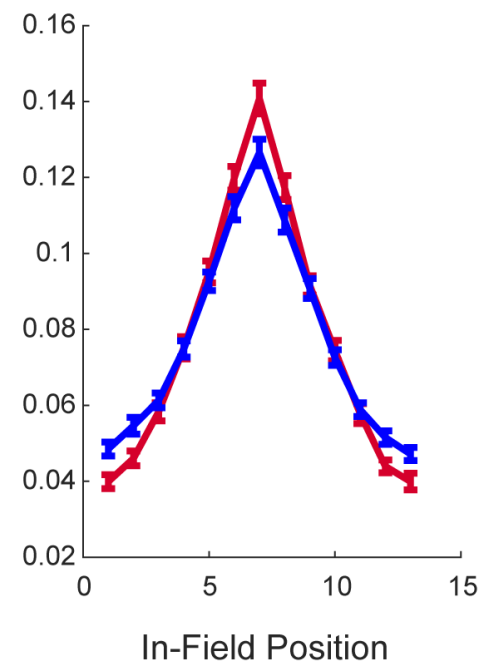

Figure S11: In-field firing rate probability as a function of gamma coefficient. (A) Normalized spike probability within the cell's place field for single spikes, for different gamma coefficients (blue for slow gamma; red for medium gamma). (B) Normalized spike probability within the cell's place field for burst spikes, for different gamma coefficients (blue for slow gamma; red for medium gamma). 
Single Spikes (All Cells)

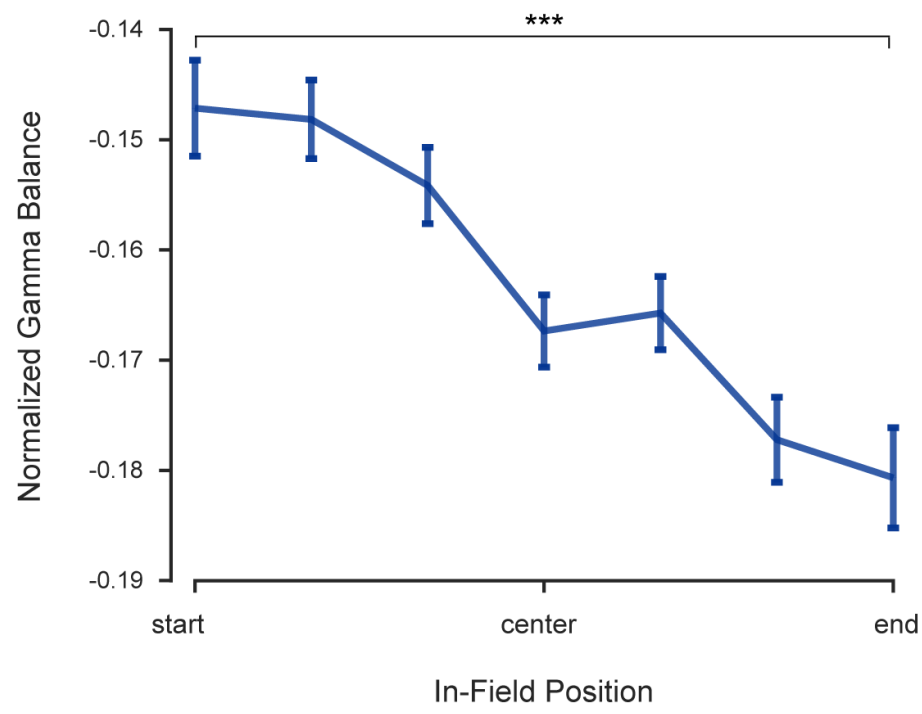

Figure S12: Instantaneous medium/slow gamma balance as a function of in field position. Normalized gamma balance (medium gamma vs slow gamma) within the cell's place field. Values at the start and the end of the place field are statistically different ( $\mathrm{p}<0.01$; two-sample t-test). 